\title{
WEIGHTED BIHARMONIC GREEN FUNCTIONS FOR RATIONAL WEIGHTS
}

\author{
MIROSLAV ENGLIŠ \\ Mathematical Institute of the Academy of Sciences, Žitná 25, 11567 Prague 1, Czech Republic \\ E-mail:englis@math.cas.cz
}

(Received 11 July, 1997)

\begin{abstract}
We present an algorithm for computing the Green function of the weighted biharmonic operator $\Delta\left|P^{\prime}\right|^{-2} \Delta$ on the unit disc (with Dirichlet boundary conditions) for rational functions $P$. As an application, we show that if $P$ is a Blaschke product with two zeros $\alpha_{1}, \alpha_{2}$ the Green function is positive if and only if $\left|\left(\alpha_{1}-\alpha_{2}\right) /\left(1-\bar{\alpha}_{1} \alpha_{2}\right)\right| \leq \frac{2}{7} \sqrt{10}$, and also obtain an explicit formula for the Green function of the operator $\Delta|G|^{-2} \Delta$, where $G$ is the canonical zero-divisor of a finite zero set on the Bergman space.
\end{abstract}

0. Introduction. Let $\mathbf{D}$ be the unit disc in the complex plane $\mathbf{C}$ and $w \in \mathbf{C}^{\infty}(\overline{\mathbf{D}})$ a function positive on the closed disc $\overline{\mathbf{D}}$ except for a finite set $S$ of singularities inside $\mathbf{D}$, where it is allowed to have a zero; such functions will be termed weights. We will be interested in the weighted biharmonic operators $\Delta w^{-1} \Delta$, where $\Delta$ is the Laplacian. For $f \in C^{\infty}(\overline{\mathbf{D}})$, a function $v$ is said to be a solution to the equation $\Delta w^{-1} \Delta v=f$ if $v \in W_{2}(\mathbf{D})$, the second-order Sobolev space on $\mathbf{D}$, and there exists a function $F \in W_{2}$ (D) such that $\Delta F=f$ and $w F=\Delta v$. It follows by the standard elliptic regularity theorems that in fact $F \in C^{\infty}(\mathbf{D})$ and $v \in C^{\infty}(\mathbf{D})$. The Green function $u(x, y)$ for the operator $\Delta w^{-1} \Delta$ is the solution to the boundary value problem

$$
\begin{cases}\Delta_{x} w(x)^{-1} \Delta_{x} u(x, y)=\delta(x-y) & (\delta \text { is the delta function }) \\ u(x, y)=\nabla_{x} u(x, y)=0 & \text { for } x \in \partial \mathbf{D}\end{cases}
$$

for each fixed $y \in \mathbf{D}$. This must again be understood in the sense that $u_{y}=u(\cdot, y)$ belongs to $W_{2}^{0}(\mathbf{D})$ and

$$
\Delta u_{y}=w\left(\Gamma(\cdot, y)+f_{y}\right),
$$

where $\Gamma(x, y)$ is the ordinary Green function for the Laplace operator $\Delta$ and $f_{y}$ is harmonic on D. By elliptic regularity, one then has $u_{y} \in C^{\infty}(\mathbf{D} \backslash\{y\}) \cap C^{1}(\mathbf{D})$ and, as $\Delta w^{-1} \Delta$ is properly elliptic on any domain obtained from $\overline{\mathbf{D}}$ upon deleting sufficiently small discs around each point in $S$, also $u_{y} \in C^{\infty}(\overline{\mathbf{D}} \backslash(S \cup\{y\}))[\mathbf{6}$, Theorem 7.36]; thus in fact

$$
u(\cdot, y) \in C^{\infty}(\overline{\mathbf{D}} \backslash\{y\}) \cap C^{1}(\overline{\mathbf{D}}),
$$

and also the function $f_{y}$ in $(0.2)$ belongs to $C^{\infty}(\overline{\mathbf{D}})$. Standard arguments show that the Green function, if it exists, indeed does the job we expect it to do; that is, for $f \in C^{\infty}(\overline{\mathbf{D}})$ the convolution with $u$ gives the solution $v \in C^{\infty}(\overline{\mathbf{D}})$ to the equation $\Delta w^{-1} \Delta v=f$ that vanishes on the boundary together with its first order derivatives; this justifies the name. 
Quite recently, a lot of attention has been attracted by the problem of positivity of these Green functions, for various weights $w$. The motivation for this comes mainly from applications in the theory, initiated by Hedenmalm, of contractive zero-divisors and factorization of functions in Bergman spaces; cf. [2], [3], [8]. Namely, for a finite sequence $\alpha=\left\{\alpha_{1}, \ldots, \alpha_{n}\right\}$ of points in $\mathbf{D}$, let $\mathcal{N}=\mathcal{N}_{\alpha}$ be the subspace of the Bergman space $A^{2}(\mathbf{D})=\left\{f \in L^{2}(\mathbf{D}): f\right.$ is holomorphic on $\left.\mathbf{D}\right\}$ consisting of functions that vanish at $\alpha_{1}, \ldots, \alpha_{n}$ (counting multiplicities), and $G=G_{\boldsymbol{\alpha}}$ the function in the unit ball of $\mathcal{N}$ which maximizes $\operatorname{Re} f(0)$ among all $f \in \mathcal{N}$ with $\|f\| \leq 1$ :

$$
G \in \mathcal{N},\|G\| \leq 1, \quad \operatorname{Re} G(0)=\max \{\operatorname{Re} f(0): f \in \mathcal{N},\|f\| \leq 1\}
$$

(If some of the points $\alpha_{i}$ coincide with the origin, this has to be modified by taking instead $\operatorname{Re} f^{(m)}(0)$, where $m$ is the number of occurrences of 0 in the sequence $\alpha_{1}, \ldots, \alpha_{n}$.) It turns out that these functions, called extremal functions of the zero sets $\alpha$, share many properties characteristic for the Blaschke products in the Hardy space $H^{2}$ on the unit circle. More precisely, the function $G$ vanishes precisely at $\alpha_{1}, \ldots, \alpha_{n}$ in $\overline{\mathbf{D}}$ (counting multiplicities), and for any $f \in \mathcal{N}$ one has $f / G \in A^{2}(\mathbf{D})$ and

$$
\|f / G\| \leq\|f\|
$$

that is, $G$ is a contractive divisor of $\mathcal{N}$ into $A^{2}(\mathbf{D})$. Of course, one cannot expect the analogy to go very far. For instance, the $\leq$ sign cannot be replaced by equality in (0.5), and also the assignment $\alpha \mapsto G_{\alpha}$ is not multiplicative; i.e. it is not true that $G_{\alpha \cup \beta}=G_{\alpha} G_{\beta}$. However, one can still hope for a somewhat weaker property than multiplicativity of $G_{\alpha}$ to be valid, namely, the domination property. The latter means that for any zero-set $\alpha$ which is contained in another zero-set $\beta$, it is true that for all $f \in \mathcal{N}_{\beta}$ we have

$$
\left\|f / G_{\beta}\right\| \leq\left\|f / G_{\alpha}\right\|
$$

The original proof of $(0.5)$ by Hedenmalm proceeded via an involved explicit calculation with power series. Later, a simpler proof was found by Duren, Khavinson, Shapiro and Sundberg [2] which established (0.5) as a consequence of the fact that the Green function for the biharmonic operator $\Delta^{2}$ on $\mathbf{D}$ is positive. By a slight modification [8] their argument also shows that (0.6) holds for a given finite zero-set $\alpha$ and any finite zero-set $\beta \supset \alpha$ provided that the Green function for the weighted biharmonic operator $\Delta\left|G_{\alpha}\right|^{-2} \Delta$ on $\mathbf{D}$ is positive. With minor modifications, the whole theory also extends to weighted Bergman spaces $A^{2}(\mathbf{D}, \rho)=$ $\left\{f \in L^{2}(\mathbf{D}, \rho): f\right.$ is holomorphic on $\left.\mathbf{D}\right\}$ with suitable weight functions $\rho$. Here "suitable" means, roughly, that (i) the space $A^{2}(\mathbf{D}, \rho)$ contains all polynomials, (ii) they are dense in it, (iii) the reproducing kernels $K(\cdot, y)$ of $A^{2}(\mathbf{D}, \rho)$ extended to a continuous function on the closed disc $\overline{\mathbf{D}}$ for each $y \in \mathbf{D}$, (iv) $K(\cdot, 0)$ does not vanish in $\overline{\mathbf{D}}$, and (v) for a zero set $\alpha$ consisting of a single point, the corresponding extremal function $G_{\alpha}$ (defined again as the solution of the extremal problem (0.4), but with the norms being taken in $A^{2}(\mathbf{D}, \rho)$ ) vanishes at that point only and nowhere else in D. Of course, (i) is just equivalent to $\rho$ being integrable over D; (ii)-(iv) are automatically fulfilled if $\rho$ is radial, i.e. depends only on the modulus $|z|$; and (v) is known to hold whenever $\log \rho$ is subharmonic, and also, for instance, for 
$\rho(z)=\left(1-|z|^{2}\right)^{q}$ for $0<q \leq 1$ (but not for $q>1$ ). An argument as in [2] then shows that, if the Green function for the operator $\Delta\left(\rho|K(\cdot, 0)|^{2}\right)^{-1} \Delta$ on $\mathbf{D}$ is positive, (i)-(v) already imply that $G_{\alpha}$ vanishes precisely on $\alpha$ in $\mathbf{D}$ for any finite zero-set $\boldsymbol{\alpha}$, that it extends continuously to $\overline{\mathbf{D}}$ and has no zeros on $\partial \mathbf{D}$, and that an analogue of (0.5) holds on $A^{2}(\mathbf{D}, \rho)$. The contractive divisor is then $G_{\alpha}(z) \sqrt{K(0,0)} / K(z, 0)$, which reduces to a constant multiple of $G_{\alpha}$ if the weight $\rho$ is a radial function. Similarly, modifying the procedure in [8] in an obvious way, it can likewise be shown that (0.6) holds for a given finite zero-set $\alpha$ and any finite zero-set $\beta \supset \alpha$ on $A^{2}(\mathbf{D}, \rho)$ with "suitable" weight $\rho$ provided the Green function for the weighted biharmonic operator

$$
\Delta\left(\rho\left|G_{\alpha}\right|^{2}\right)^{-1} \Delta
$$

on $\mathbf{D}$ is positive. (Taking $\alpha$ to be the empty set, $G_{\emptyset}=K(\cdot, 0) / \sqrt{K(0,0)}$, and so we recover the previous operator $\Delta\left(\rho|K(\cdot, 0)|^{2}\right)^{-1} \Delta$ as a special case.)

For the unweighted biharmonic operator $\Delta^{2}$ on a domain $\Omega$ in the plane, the question of positivity of the Green function is of an old date. In that case, the Green function is related to bending of a clamped plate of the shape of $\Omega$ and to creeping flow on $\Omega$, and the question concerning its positivity comes back to Hadamard and Boggio (see the discussion in [9] for more details and references); the answer is known to be affirmative for a few domains (the disc, Pascal's limaçon, images of D under polynomial conformal maps $\phi$ that are (coefficientwise) sufficiently close to the identity [16]) and negative for many others (sufficiently eccentric ellipses, sufficiently elongated rectangles, etc.; if we allow $\Omega$ to be unbounded or multiply connected, we can also add the infinite strip and all annuli [5]). Observe that if $\phi$ is a conformal map of $\mathbf{D}$ onto a simply connected $\Omega$, the pullback via $\phi$ of the Green function for $\Delta^{2}$ on $\Omega$ is the Green function for $\Delta\left|\phi^{\prime}\right|^{-2} \Delta$ on $\mathbf{D}$; thus, some of the results just mentioned can also be interpreted as results about weighted biharmonic operators $\Delta\left|\phi^{\prime}\right|^{-2} \Delta$ on $\mathbf{D}$. For radial weights $w$, a number of results have been obtained by Shimorin [12], [13], [14] and Hedenmalm [8], [10] for $w(z)=\left(1-|z|^{2}\right)^{q}$, $-1<q \leq 1$, and by Shimorin [15], for $w$ radial, integrable, log-subharmonic and sufficiently smooth. However, apart from the computation of Hedenmalm [9] for $w=|z|^{2 q}, q>-1$, and his formula (containing a small error) for the Green function of $\Delta\left|G_{\alpha}\right|^{-2} \Delta$ for $\rho \equiv 1$ and $\alpha$ consisting of a single point in $\mathbf{D}[\mathbf{8}]$, to this day nothing seems to be known about the weighted biharmonic Green functions and their positivity for weights of the form $w=|f|^{2}$ with $f$ a holomorphic function which vanishes somewhere in D. In particular, the question of the positivity of the Green function for the operator $(0.7)$ even in the simplest unweighted case $\rho \equiv 1$ remains open at present.

In this paper we will consider weighted biharmonic operators on $\mathbf{D}$ of the form $\Delta\left|P^{\prime}\right|^{-2} \Delta$, where $P$ is a rational function. We present an algorithm which, for a given $P$, makes it possible to calculate the corresponding Green function explicitly; more precisely, the calculation is reduced to solving a system of $m$ linear equations for certain coefficients, where $m$ is the number of points $\alpha$ in $\mathbf{D}$ for which either $P^{\prime}(\alpha)=0$ or $P(1 / \bar{\alpha})$ is a pole (multiple zeros and poles being counted in an appropriate way). Further, we can rigorously prove the existence of the Green function for any rational $P$. Note that for the operator $\Delta\left|P^{\prime}\right|^{-2} \Delta$, being singular and hence not elliptic in general, this does not readily follow from general PDE theory. All this is done in Section 1. As a first application we consider in Section 2 the case in which 
$P$ is a Blaschke product, and derive an explicit formula for the Green function if $P$ is of length two (Theorem 2.4); it turns out that the Green function is positive if and only if the hyperbolic distance of the two zeros does not exceed $\frac{2}{7} \sqrt{10}$. It is conceivable that for a general Blaschke product $P$ the corresponding Green function is positive if and only if the zeros of $P$ are not spaced "too widely apart" in $\mathbf{D}$. The third section is devoted to the most interesting case of the operators $\Delta\left|G_{\alpha}\right|^{-2} \Delta$, where $G_{\boldsymbol{\alpha}}$ are the extremal functions of finite zero-sets $\alpha$ introduced above $(\rho \equiv 1)$. We even consider their more general analogues obtained upon replacing $\operatorname{Re} f(0)$ by $\operatorname{Re} f\left(\alpha_{0}\right)$ in (0.4), for some $\alpha_{0} \in \mathbf{D}$. Our main result is a formula for the Green function in terms of the Gramm matrix $\left(G_{j k}\right)=\left\{\left(1-\bar{\alpha}_{k} \alpha_{j}\right)^{-2}\right\}_{j, k=0}^{n}$ and its inverse $\left(A_{j k}\right)$ (Theorem 3.1); this formula also admits an interesting rephrasement in terms of Toeplitz or "model" operators (truncations of the adjoint of the Bergman shift to its invariant subspace) on $A^{2}(\mathbf{D})$. For the most part it is assumed that the points $\alpha_{0}, \ldots, \alpha_{n}$ are mutually distinct, yet the operator rephrasement just mentioned implies immediately that the formula, in fact, extends by continuity to all $\left(\alpha_{0}, \alpha_{1}, \ldots, \alpha_{n}\right) \in \mathbf{D}^{n+1}$, and further the resulting Green function depends continuously on the $\alpha_{j}$. We also establish the positivity of the Green function in the case $n=1$, for any $\alpha_{0}, \alpha_{1} \in \mathbf{D}$ (thereby extending Hedenmalm's result for $\alpha_{0}=0$ mentioned above), and for $n=2, \alpha_{0}=0$, and any $\alpha_{1}=-\alpha_{2} \in \mathbf{D}$. For $n>1$, we are unable to prove the positivity in general, but indicate that it is probably a consequence of a much stronger assertion concerning positive-definiteness of a certain quadratic form $\varrho(\xi, \xi)$ in $(n+1)^{2}$ variables $\xi_{j k}, j, k=0,1, \ldots, n$ (Conjecture 3.5) whose coefficients are associated with $G_{j k}$ and $A_{j k}$ in a fairly simple way.

Throughout the paper, it will be convenient to normalize the Lebesgue measure so that $\mathbf{D}$ has area 1 (instead of $\pi$ ) and to adopt a slightly nonstandard definition of the Laplacian which differs from the usual one by a factor of 4 . This has the advantage that the constant function one has unit norm in $A^{2}(\mathbf{D}), \Delta|f|^{2}=\left|f^{\prime}\right|^{2}$, for any holomorphic $f$, and the Green function for the operator $\Delta$ on $\mathbf{D}$ is simply

$$
\Gamma(x, y)=\log \left|\frac{x-y}{1-\bar{y} x}\right|^{2}
$$

without the factor $1 / 4 \pi$ in front.

1. Preliminaries. Let $P$ be a nonconstant function holomorphic on $\overline{\mathbf{D}}, p=P^{\prime}$ its derivative, $S=\{z \in \overline{\mathbf{D}}: p(z)=0\}$ the zeros of $p$ in $\overline{\mathbf{D}}, \partial \equiv \partial / \partial z, \bar{\partial} \equiv \partial / \partial \bar{z}$ the Wirtinger operators, $\Delta=\partial \bar{\partial}$ the Laplacian divided by 4 . We shall assume throughout that $S \subset \mathbf{D}$; i.e. that $p=P^{\prime}$ has no zeros on the boundary $\partial \mathbf{D}$ (so that $S$ is a finite set).

The Green function for the weighted biharmonic operator $\Delta|p|^{-2} \Delta$ is, as observed in the Introduction, a function $u(x, y)$ which for $y$ fixed is in $C^{1}(\overline{\mathbf{D}}) \cap C^{\infty}(\overline{\mathbf{D}} \backslash\{y\})$ and satisfies the equation

$$
\Delta_{x} u(x, y)=|p(x)|^{2}\left(\log \left|\frac{x-y}{1-\bar{y} x}\right|^{2}+2 \operatorname{Re} h_{0}(x)\right) \quad \forall x \in \mathbf{D} \backslash\{y\},
$$

for some $h_{0}$ holomorphic on $\overline{\mathbf{D}}$, and the boundary conditions

$$
u(x, y)=0 \quad \forall x \in \partial \mathbf{D},
$$




$$
\frac{\partial u}{\partial x}(x, y)=\frac{\partial u}{\partial \bar{x}}(x, y)=0 \quad \forall x \in \partial \mathbf{D} .
$$

The function $h_{0}$ depends on $y$.

Introduce the notation

$$
\begin{gathered}
\mathcal{P}(x)=P(x)-P(y), \\
\zeta(x)=\frac{(x-y)(1-\bar{y} x)}{1-|y|^{2}} .
\end{gathered}
$$

Again these functions depend on $y$ even though this fact is not explicitly reflected by the notation.

Let $\mathcal{O}$ stand for the set of functions holomorphic on the closed disc $\overline{\mathbf{D}}$; (that is, holomorphic on $\mathbf{D}$ and $C^{\infty}$ on $\overline{\mathbf{D}}$ ).

Proposition 1.1 If the Green function (1.1) exists, then for each $y \in \mathbf{D}$ there are functions $H$ and holomorphic on $\overline{\mathbf{D}}$ such that

$$
u(x, y)=|\mathcal{P}(x)|^{2} \log \left|\frac{x-y}{1-\bar{y} x}\right|^{2}+2 \operatorname{Re}[h(x)-\bar{p}(x) H(x)] \quad \forall x \in \overline{\mathbf{D}} .
$$

The functions $H$ and $h$ are uniquely determined up to a transformation

$$
H \mapsto H+\eta+i \theta \mathcal{P}, h \mapsto h+i \xi+\bar{\eta} \mathcal{P}, \eta \in \mathbf{C}, \xi, \theta \in \mathbf{R},
$$

and satisfy

$$
\begin{gathered}
\operatorname{Re}[h-\overline{\mathcal{P}} H]=0 \quad \text { on } \partial \mathbf{D}, \\
\frac{|\mathcal{P}|^{2}}{\zeta}-\overline{\mathcal{P}} H^{\prime}-p \bar{H}+h^{\prime}=0 \quad \text { on } \partial \mathbf{D}, \\
\frac{\mathcal{P} / \zeta-H^{\prime}}{p} \in \mathcal{O} .
\end{gathered}
$$

Conversely, for any two functions $H, h$ satisfying (1.6)-(1.8), (1.4) is the Green function for $\Delta|p|^{-2} \Delta$.

Proof. Let $H$ be a primitive to the (holomorphic) function $\mathcal{P} / \zeta-p h_{0}$, where $h_{0}$ is the function appearing in (1.1). Since

$$
\begin{gathered}
\Delta_{x}|\mathcal{P}|^{2} \log \left|\frac{x-y}{1-\bar{y} x}\right|^{2}=|p|^{2} \log \left|\frac{x-y}{1-\bar{y} x}\right|^{2}+2 \operatorname{Re}[\bar{p} \mathcal{P} / \zeta], \\
\Delta(\mathcal{P} \bar{H}+\overline{\mathcal{P}} H)=2 \operatorname{Re}\left[\frac{\bar{p} \mathcal{P}}{\zeta}-|p|^{2} h_{0}\right],
\end{gathered}
$$

we see that the difference of $u(x, y)$ and $|\mathcal{P}|^{2} \log \left|\frac{x-y}{1-\bar{y} x}\right|^{2}-(\mathcal{P} \bar{H}+\overline{\mathcal{P}} H)$ must be a harmonic function, and hence of the form $h+\bar{h}$ for some holomorphic function $h$. This proves the existence. The uniqueness claim is straightforward. 
Since for $x \in \partial \mathbf{D}$

$$
\begin{gathered}
u(x, y)=2 \operatorname{Re}[h-\overline{\mathcal{P}} H], \\
\frac{\partial u}{\partial x}(x, y)=\frac{|\mathcal{P}|^{2}}{\zeta}-\overline{\mathcal{P}} H^{\prime}-p \bar{H}+h^{\prime}, \\
\frac{\partial u}{\partial \bar{x}}=\frac{\overline{\partial u}}{\partial x} \text { (since (1.4) is real-valued), }
\end{gathered}
$$

the equalities (1.6) and (1.7) follow from the boundary conditions (1.2) and (1.3). Also (1.8) is satisfied as $\mathcal{P} / \zeta-H^{\prime}=p h_{0}$, in view of the definition of $H$.

The converse part is immediate from (1.9) and (1.10).

Of course, the functions $H$ and $h$ again depend on $y$.

We remark that it is immediate from (1.4) that for $y$ fixed $u(x, y)$ is in fact realanalytic on $\overline{\mathbf{D}} \backslash\{y\}$.

Let $\mathbf{G}=\mathbf{C} \cup\{\infty\}$ denote the Gauss sphere. For a meromorphic function $f$ and $\alpha \in \mathbf{G}$ we denote by pmult $(f ; a)$ the multiplicity of the pole of $f$ at $a(\operatorname{pmult}(f ; a)=0$ if there is no pole); similarly zmult $(f ; a)=\operatorname{pmult}(1 / f ; a)$ denotes the multiplicity of the zero at $a$.

Proposition 1.2 Suppose that the Green function (1.1) exists and that $P$ is a rational function. Then $H$ and $h$ are also rational functions and $\forall z \in \mathbf{G} \backslash \mathbf{D}$,

$$
\begin{aligned}
\operatorname{pmult}(H ; z) & \leq \max (\operatorname{pmult}(P ; z), \operatorname{zmult}(p, 1 / \bar{z})), \\
\operatorname{pmult}(h ; z) & \leq \max (\operatorname{pmult}(P ; z), \operatorname{zmult}(p, 1 / \bar{z})) .
\end{aligned}
$$

Proof. Define

$$
K(z)=\overline{H(1 / \bar{z})}, \quad \mathcal{Q}(z)=\overline{\mathcal{P}(1 / \bar{z})}, \quad \tilde{h}(z)=\overline{h(1 / \bar{z})} .
$$

These are holomorphic functions on $\mathbf{G} \backslash \mathbf{D}$; on the unit circle we have

$$
K(z)=\overline{H(z)}, \quad \mathcal{Q}(z)=\overline{\mathcal{P}(z)}, \quad \tilde{h}(z)=\overline{h(z)} .
$$

The conditions (1.6) and (1.7) can thus be rewritten

$$
\begin{gathered}
h+\tilde{h}=\mathcal{Q} H+\mathcal{P} K \quad \text { on } \partial \mathbf{D}, \\
p K=\frac{\mathcal{P} \mathcal{Q}}{\zeta}-\mathcal{Q} H^{\prime}+h^{\prime} \quad \text { on } \partial \mathbf{D} .
\end{gathered}
$$

The LHS of the last equality is a function meromorphic on $\mathbf{G} \backslash \mathbf{D}$, while the RHS is meromorphic on $\overline{\mathbf{D}}$; it follows that both sides extend to meromorphic functions on G; that is, rational functions. Hence (1.15) prevails on all of G. By (1.8), (1.15) can be rewritten as

$$
K=\mathcal{Q} h_{0}+h^{\prime} / p \quad \forall z \in \overline{\mathbf{D}}
$$


with $h_{0}$ holomorphic on $\overline{\mathbf{D}}$, and it follows that

$$
\operatorname{pmult}(K ; z) \leq \max (\operatorname{pmult}(\mathcal{Q} ; z), \operatorname{zmult}(p, z)) \quad \forall z \in \overline{\mathbf{D}}
$$

Passing back to $H$ via (1.12) gives the first inequality in (1.11). This settles $H$. From (1.15), we now see that $h^{\prime}$ is also a rational function. Write

$$
h^{\prime}(z)=\sum_{j} \frac{c_{j}}{z-z_{j}}+r(z)
$$

where $r(z)$ has only poles of order at least two and $z_{j}, j=1,2, \ldots$, are the simple poles of $h^{\prime}$ indexed so that $1<\left|z_{1}\right| \leq\left|z_{2}\right| \leq \ldots$. Let $\Omega$ be the domain obtained by deleting from $\mathbf{G}$ the line segments $\left[z_{j}, \infty\right]$ and $\left[0,1 / \bar{z}_{j}\right](j=1,2, \ldots)$. On $\Omega$ we have

$$
\begin{aligned}
& h(z)=\sum_{j} c_{j} \log \left(z-z_{j}\right)+R(z), \\
& \tilde{h}(z)=\sum_{j} \bar{c}_{j} \log \frac{1-\bar{z}_{j} z}{z}+\tilde{R}(z),
\end{aligned}
$$

where $R(z)$ and $\tilde{R}(z):=\overline{R(1 / \bar{z})}$ are rational functions. In view of (1.14) it follows that

$$
S(z) \equiv \sum_{j} c_{j} \log \left(z-z_{j}\right)+\bar{c}_{j} \log \frac{1-\bar{z}_{j} z}{z} \text { defines a rational function } S .
$$

If $c_{1} \neq 0$, then as $z \rightarrow z_{1}, S(z) \rightarrow \infty$ and $\left(z-z_{1}\right) S(z) \rightarrow 0$; this cannot happen for a rational function. Thus $c_{1}=0$. Proceeding by induction we show that all $c_{j}=0$, so that $h^{\prime}$ has no simple poles and $h$ is a rational function as well. By uniqueness, (1.14) prevails on all of $\mathbf{G}$; consequently, for any $z \in \mathbf{G} \backslash \mathbf{D}$ we have

$\operatorname{pmult}(h ; z)=\operatorname{pmult}(h+\tilde{h} ; z)=\operatorname{pmult}(\mathcal{P} K+\mathcal{Q} H ; z) \leq \max (\operatorname{pmult}(\mathcal{P} ; z), \operatorname{pmult}(H ; z))$

and the second claim in (1.11) follows.

THEOREM 1.3 Let $P$ be a nonconstant rational function with no poles in $\overline{\mathbf{D}}$. Then the Green function for the weighted biharmonic operator $\Delta\left|P^{\prime}\right|^{-2} \Delta$ exists.

Proof. By Proposition 1.1, it suffices to find (for each y) $H \in \mathcal{O}$ such that, in the notation (1.12),

$$
\frac{\mathcal{P} / \zeta-H^{\prime}}{p} \in \mathcal{O} \text { and } p K+\mathcal{Q} H^{\prime}-\frac{\mathcal{P Q}}{\zeta} \in \mathcal{O}
$$

Indeed, let $h$ be the holomorphic primitive to $p K+\mathcal{Q} H^{\prime}-\mathcal{P} \mathcal{Q} / \zeta$ on $\overline{\mathbf{D}}$, normalized so that

$$
\operatorname{Re} h(1)=\operatorname{Re}[\overline{\mathcal{P}}(1) H(1)] .
$$

Then (1.7) and (1.8) hold, and by the equalities (1.10), $\operatorname{Re}[h-\overline{\mathcal{P}} H]$ is constant on dD. In view of (1.17), this constant must be zero, so that (1.6) is satisfied as well, and the existence of the Green function follows by Proposition 1.1. 
By Proposition 1.2, it further suffices to look for $H$ among rational functions satisfying (1.11); adjusting the $\eta$ in (1.5) if necessary, we may also assume that $H(y)=0$.

For simplicity, we treat in detail only the case when $P$ has only simple poles in $\mathbf{G}$ and $p=P^{\prime}$ has only simple zeros in $\mathbf{D}$. The general case involves no new ideas, only more technicalities.

Thus let $\alpha_{1}, \alpha_{2}, \ldots, \alpha_{N}$ be all the points $z$ in $\mathbf{D}$ for which either $p(z)=0$ or $P(1 / \bar{z})=\infty$; we can assume that $\alpha_{1}, \ldots, \alpha_{n}$ are all the zeros of $p$ in $\mathbf{D}$ and $1 / \bar{\alpha}_{M+1}, \ldots, 1 / \bar{\alpha}_{N}$ are all the poles of $P$ in $\mathbf{G}(0 \leq M \leq n \leq N)$; clearly $N \geq 1$ as $P$ is nonconstant. The function $\mathcal{P}(z)=P(z)-P(y)$ has the same poles as $P$ and, further, a zero at $y$; it follows that it is of the form

$$
\mathcal{P}(z)=\sum_{j=1}^{N} \frac{a_{j}(z-y)}{\left(1-\bar{\alpha}_{j} z\right)\left(1-\bar{\alpha}_{j} y\right)},
$$

for some numbers $a_{j}$ (depending on $y$ ), where

$$
\begin{gathered}
a_{j}=0, \quad j=1,2, \ldots, M, \\
a_{j} \neq 0, \quad j=M+1, \ldots, N .
\end{gathered}
$$

In view of the observation in the penultimate paragraph, we shall look for $H$ in the form

$$
H(z)=\sum_{j=1}^{N} \frac{s_{j}(z-y)}{\left(1-\bar{\alpha}_{j} z\right)\left(1-\bar{\alpha}_{j} y\right)},
$$

where $s_{j}$ are coefficients to be determined. Observe that

$$
H^{\prime}(z)=\sum_{j=1}^{N} \frac{s_{j}}{\left(1-\bar{\alpha}_{j} z\right)^{2}} .
$$

Introduce the quantities

$$
R_{k}:=\frac{\mathcal{P}\left(\alpha_{k}\right)}{\zeta\left(\alpha_{k}\right)}=\frac{1-|y|^{2}}{1-\bar{y} \alpha_{k}} \sum_{j=1}^{N} \frac{a_{j}}{\left(1-\bar{\alpha}_{j} \alpha_{k}\right)\left(1-\bar{\alpha}_{j} y\right)} .
$$

Since all the zeros of $p$ in $\mathbf{D}$ are assumed to be simple, the first condition in (1.16) takes the simple form

$$
H^{\prime}\left(\alpha_{k}\right)=R_{k}(k=1, \ldots, n)
$$

that is, by $(1.20)$,

$$
\sum_{j=1}^{N} G_{k j} s_{j}=R_{k}(k=1, \ldots, n),
$$

where we have introduced the matrix 


$$
G_{k j}:=\left(1-\bar{\alpha}_{j} \alpha_{k}\right)^{-2} \text {. }
$$

The only possible poles of $K$ and $\mathcal{Q}$ in $\mathbf{D}$ are at $z=\alpha_{k}(k=1, \ldots, N)$; thus the second condition in (1.16) is equivalent to

$$
\lim _{z \rightarrow \alpha_{k}}\left(z-\alpha_{k}\right)\left(p K+\mathcal{Q} H^{\prime}-\mathcal{P} \mathcal{Q} / \zeta\right)=0 \quad(k=1, \ldots, N) .
$$

Since

$$
\lim _{z \rightarrow \alpha_{k}}\left(z-\alpha_{k}\right) K(z)=\bar{s}_{k}, \quad \lim _{z \rightarrow \alpha_{k}}\left(z-\alpha_{k}\right) \mathcal{Q}(z)=\bar{a}_{k},
$$

this gives

$$
p\left(\alpha_{k}\right) \bar{s}_{k}+\bar{a}_{k} H^{\prime}\left(\alpha_{k}\right)=\bar{a}_{k} R_{k}
$$

or

$$
p\left(\alpha_{k}\right) \bar{s}_{k}+\bar{a}_{k} \sum_{j=1}^{N} G_{k j} s_{j}=\bar{a}_{k} R_{k} \quad(k=1, \ldots, N) .
$$

For $k=1, \ldots, n$ these equalities follow from (1.22), since $p\left(\alpha_{k}\right)=0$; thus we need to consider them only for $n<k \leq N$.

Consider quite generally the system

$$
\begin{gathered}
\sum_{j=1}^{N} G_{k j} s_{j}=R_{k} \quad(k=1, \ldots, n), \\
\bar{a}_{k}^{-1} p\left(\alpha_{k}\right) \sigma_{k}+\sum_{j=1}^{N} G_{k j} s_{j}=R_{k} \quad(k=n+1, \ldots, N), \\
\sum_{j=1}^{N} \bar{G}_{k j} \sigma_{j}=\bar{R}_{k} \quad(k=1, \ldots, n), \\
a_{k}^{-1} \overline{p\left(\alpha_{k}\right)} s_{k}+\sum_{j=1}^{N} \bar{G}_{k j} \sigma_{j}=\bar{R}_{k} \quad(k=n+1, \ldots, N),
\end{gathered}
$$

of $2 N$ linear equations in $2 N$ unknowns $s_{1}, \ldots, s_{N}, \sigma_{1}, \ldots, \sigma_{N}$. Assume that we know that all solutions of the corresponding homogenous system

$$
\begin{aligned}
& \sum_{j=1}^{N} G_{k j} s_{j}=0(k=1, \ldots, n), \\
& \bar{a}_{k}^{-1} p\left(\alpha_{k}\right) \sigma_{k}+\sum_{j=1}^{N} G_{k j} s_{j}=0(k=n+1, \ldots, N), \\
& \sum_{j=1}^{N} \bar{G}_{k j} \sigma_{j}=0 \quad(k=1, \ldots, n), \\
& a_{k}^{-1} \overline{p\left(\alpha_{k}\right)} s_{k}+\sum_{j=1}^{N} \bar{G}_{k j} \sigma_{j}=0 \quad(k=n+1, \ldots, N),
\end{aligned}
$$


are given by

$$
s_{j}=c a_{j}, \sigma_{j}=-c \bar{a}_{j},(c \in \mathbf{C})
$$

By a familiar theorem of linear algebra, it will then follow that the system (1.25) is solvable if and only if

$$
\sum_{j=1}^{N} \overline{c a_{j}} R_{j}+\sum_{j=1}^{N}\left(-\bar{c} a_{j}\right) \bar{R}_{j}=0 \quad \forall c \in \mathbf{C} ; \text { i.e. } \sum_{j=1}^{N} a_{j} \bar{R}_{j} \in \mathbf{R},
$$

and in that case a solution will exist that satisfies $\sigma_{k}=\bar{s}_{k}$ and will be unique up to a transformation $s_{k} \mapsto s_{k}+i \theta a_{k}, \sigma_{k} \mapsto \sigma_{k}-i \theta \bar{a}_{k}(\theta \in \mathbf{R})$. As in our case

$$
\sum_{k=1}^{N} a_{k} \bar{R}_{k}=\sum_{j, k=1}^{N} \frac{\left(1-|y|^{2}\right) a_{k} \bar{a}_{j}}{\left(1-\bar{\alpha}_{k} y\right)\left(1-\bar{y} \alpha_{j}\right)\left(1-\bar{\alpha}_{k} \alpha_{j}\right)}
$$

is indeed real (even positive), we shall thus obtain a solution to (1.22) and (1.24), and hence also the function $H$, which will be unique up to a transformation $H \mapsto H+i \theta \mathcal{P}$, in agreement with (1.5); this will complete the proof.

Now suppose that $(s, \sigma)$ is a solution to the homogenous system (1.26). Let $H$ be the corresponding function given by (1.19), $\hat{H}$ the analogous function with $\bar{\sigma}_{j}$ in the place of $s_{j}$, and $K$ and $\hat{K}$ the corresponding functions associated to $H$ and $\hat{H}$ by (1.12). Reversing the procedure used above to obtain (1.22) and (1.24), it is readily seen that the system (1.26) is equivalent to four conditions

$$
\begin{aligned}
H^{\prime} / p & \in \mathcal{O}, \\
p \hat{K}+\mathcal{Q} H^{\prime} & \in \mathcal{O}, \\
\hat{H}^{\prime} / p & \in \mathcal{O}, \\
p K+\mathcal{Q} \hat{H}^{\prime} & \in \mathcal{O} .
\end{aligned}
$$

Let $h$ and $\hat{h}$ be holomorphic primitives to $p \hat{K}+\mathcal{Q} H^{\prime}$ and $p K+\mathcal{Q} \hat{H}^{\prime}$, respectively, normalized so that the function

$$
v=\mathcal{P} \overline{\hat{H}}+\overline{\mathcal{P}} H-h-\overline{\hat{h}}
$$

vanished at $z=1$. In view of the second and fourth conditions in (1.28), we have $\partial v=\bar{\partial} v=0$ on $\partial \mathbf{D}$; it follows that $v$ is constant on $\partial \mathbf{D}$, and owing to the normalization this constant must be zero. Thus

$$
v, \nabla v=0 \quad \text { on } \partial \mathbf{D} \text {. }
$$

Let $H^{\prime}=p f$ and $\hat{H}^{\prime}=p \hat{f}$, with $f, \hat{f} \in \mathcal{O}$. Then, by Green's formula and (1.29), we have

$$
0=\int_{\mathbf{D}} \Delta v \cdot(\hat{f}+\bar{f})=\int_{\mathbf{D}}|p|^{2} \cdot|\hat{f}+\bar{f}|^{2}
$$


since $\Delta v=p \overline{\hat{H}}^{\prime}+\bar{p} H^{\prime}=|p|^{2}(\overline{\hat{f}}+f)$. It follows that $\hat{f}+\bar{f}=0$ or $f=-\overline{\hat{f}}=c \mathbf{1}$, for some complex number $c$. This means that $H^{\prime}=c p, H^{\prime}=-\bar{c} p$ and $H=c \mathcal{P}, \hat{H}=-\bar{c} \mathcal{P}$, so that $s=c a, \bar{\sigma}=-\bar{c} a$, and (1.27) follows. This completes the proof.

In the general case when $P$ is allowed to have higher-order poles and $p$ multiple zeros in $\mathbf{D}$, the equations (1.22) and (1.24) and the system (1.25) will get somewhat more complicated, since the higher derivatives of $p$ and $\mathcal{P} / \zeta$ at various $\alpha_{k}$ enter the picture; however, the reduction of the corresponding homogenous system to the conditions (1.28) as well as the subsequent argument with the function $v$ work without change so that the only solutions to the homogenous system are again given by (1.27) and the solvability of the corresponding non-homogenous system follows by a lengthy yet routine calculation. As we won't need this case in the sequel, we skip the details and leave them to the interested reader.

Recall that the Green function for the ordinary (unweighted) biharmonic operator $\Delta^{2}$ is given by the formula

$$
U(x, y)=\left[|x-y|^{2} \log \left|\frac{x-y}{1-\bar{y} x}\right|^{2}+\left(1-|x|^{2}\right)\left(1-|y|^{2}\right)\right]
$$

see e.g. [7, p. 272].

Proposition 1.4. Let $P$ be a nonconstant rational function holomorphic on $\overline{\mathbf{D}}$. For $\gamma \in \mathbf{D}$ denote

$$
\kappa(\gamma):=\max (\operatorname{pmult}(P ; 1 / \bar{\gamma}), \operatorname{zmult}(p ; \gamma))
$$

and let $N=\sum_{\gamma \in \mathbf{D}} \kappa(\gamma)$; (this is a finite sum). Then the Green function for the weighted biharmonic operator $\Delta\left|P^{\prime}\right|^{-2} \Delta$ is of the form

$$
u(z, y)=\left|\frac{P(z)-P(y)}{z-y}\right|^{2} U(z, y)+\frac{\left(1-|z|^{2}\right)^{2}\left(1-|y|^{2}\right)^{2} R(z, \bar{z}, y, \bar{y})}{\left|\prod_{\gamma \in \mathbf{D}}(1-\bar{\gamma} z)^{\kappa(\gamma)} \prod_{\gamma \in \mathbf{D}}(1-\bar{\gamma} y)^{\kappa(\gamma)}\right|^{2}},
$$

where $R(z, \bar{z}, y, \bar{y})$ is a polynomial of degree at most $N-2$ in each of the indicated variables $(R \equiv 0$ if $N<2)$.

Proof. By Proposition 1.2 the functions $H, h$ in (1.4) are rational functions with no poles in $\overline{\mathbf{D}}$ and with a pole of multiplicity at most $\kappa(\gamma)$ at each $1 / \bar{\gamma}(\gamma \in \mathbf{D})$. Consequently, they can both be expressed as

$$
\frac{\text { a polynomial in } z \text { of degree at most } N}{\prod_{\gamma \in \mathbf{D}}(1-\bar{\gamma} z)^{\kappa(\gamma)}} ;
$$

(the product in the denominator has only finitely many terms different from 1 ). Since $\operatorname{pmult}(P ; 1 / \bar{\gamma}) \leq \kappa(\gamma)$, the function $\mathcal{P}$ must likewise be of this form, and $\mathcal{P} /(z-y)$ is even of the same form with the numerator of degree at most $N-1$. Substituting this information into (1.4), we see that the difference

$$
u(z, y)-\left|\frac{\mathcal{P}}{z-y}\right|^{2} U(z, y)=-\left(1-|z|^{2}\right)\left(1-|y|^{2}\right)\left|\frac{\mathcal{P}}{z-y}\right|^{2}-(\overline{\mathcal{P}} H+\mathcal{P} \bar{H})+(h+\bar{h})
$$


must be a rational function of $z, \bar{z}$ of the form

$$
\frac{P_{2}(z, \bar{z})}{\left|\prod_{\gamma \in \mathbf{D}}(1-\bar{\gamma} z)^{\kappa(\gamma)}\right|^{2}}
$$

for some polynomial $P_{2}(z, \bar{z})$ of $z$ and $\bar{z}$ of degree at most $N$ in each of these two variables. This expression must in addition be symmetric in $y$ and $z$, since the Green functions $u$ and $U$ are, and hence so is the difference on the left-hand side of (1.30). Thus the right-hand side of (1.30) must actually be a rational function of $z, \bar{z}, y$ and $\bar{y}$ of the form

$$
\frac{P_{3}(z, \bar{z}, y, \bar{y})}{\left|\prod_{\gamma \in \mathbf{D}}(1-\bar{\gamma} z)^{\kappa(\gamma)} \prod_{\gamma \in \mathbf{D}}(1-\bar{\gamma} y)^{\kappa(\gamma)}\right|^{2}}
$$

for a polynomial $P_{3}(z, \bar{z}, y, \bar{y})$ of degree at most $N$ in each of the indicated variables. Finally, as both $u$ and $U$ vanish on the boundary (off the diagonal $z=y$ ) together with their gradients, the same must hold for the difference (1.30) and, consequently, also for the polynomial in the numerator of the last formula. Thus $P_{3}$ can be factorised as

$$
\left(1-|z|^{2}\right)^{2}\left(1-|y|^{2}\right)^{2} R(z, \bar{z}, y, \bar{y})
$$

for a certain polynomial $R$ of degree not exceeding $N-2$ in each variable. The proof is complete.

Proposition 1.5. Let $P$ be a non-constant rational function holomorphic on $\overline{\mathbf{D}}$. Assume that $P$ has only simple poles in $\mathbf{G}$ and $p=P^{\prime}$ has only simple zeros in $\mathbf{D}$. Let $\alpha_{1}, \ldots, \alpha_{N}$ be the points in $\mathbf{D}$ for which either $p(z)=0$ or $P(1 / \bar{z})=\infty$. Then the Green function for the weighted biharmonic operator $\Delta\left|P^{\prime}\right|^{-2} \Delta$ is given by

$$
u(x, y)=\left|\frac{P(x)-P(y)}{x-y}\right|^{2} U(x, y)+\left(1-|x|^{2}\right)^{2}\left(1-|y|^{2}\right)^{2} c(x, y)
$$

with

$$
\begin{aligned}
& c(x, y)=\sum_{J, K=1}^{N} \frac{e_{J K}(y, \bar{y})}{\left(1-\bar{\alpha}_{J} x\right)\left(1-\alpha_{K} \bar{x}\right)} \\
& =\sum_{J, K, L, M=1}^{N} \frac{A_{J K L M}}{\left(1-\bar{\alpha}_{J} x\right)\left(1-\alpha_{k} \bar{x}\right)\left(1-\bar{\alpha}_{L} y\right)\left(1-\alpha_{M} \bar{y}\right)},
\end{aligned}
$$

where $e_{J K}$ are rational functions of $y, \bar{y}$ and $A_{J K L M}$ are constants. Let further $a_{j}, R_{j}(j=1, \ldots, N)$ be the numbers defined by (1.18) and (1.21), respectively, and $\left(s_{j}\right) a$ solution of the system (1.22), (1.24). Then

$$
e_{J K}(y, \bar{y})=\frac{\bar{\alpha}_{J} \alpha_{K}}{\left(1-|y|^{2}\right)^{2}\left(1-\bar{\alpha}_{J} \alpha_{K}\right)^{2}}\left[\frac{\left(1-|y|^{2}\right)\left(1-\bar{\alpha}_{J} \alpha_{K}\right) a_{J} \bar{a}_{K}}{\left(1-\bar{\alpha}_{J} y\right)\left(1-\alpha_{K} \bar{y}\right)}-\left(\bar{a}_{K} S_{J}+a_{J} \bar{s}_{K}\right)\right]
$$


and $A_{J K L M}$ can be obtained by taking the limit

$$
A_{J K L M}=\lim _{\substack{y \rightarrow 1 / \alpha_{L} \\ \bar{y} \rightarrow 1 / \alpha_{M}}}\left(1-\bar{\alpha}_{L} y\right)\left(1-\alpha_{M} \bar{y}\right) e_{J K}(y, \bar{y}) .
$$

(If $L \neq M, y$ and $\bar{y}$ should be treated formally as two independent variables.) In particular,

$$
e_{J K}=0 \text { if } \alpha_{J} \alpha_{K}=0, A_{J K L M}=0 \text { if } \alpha_{J} \alpha_{K} \alpha_{L} \alpha_{M}=0 .
$$

Proof. In view of the preceding proposition the Green function is of the form (1.31) with

$$
c(x, y)=\frac{R(x, \bar{x}, y, \bar{y})}{\left|\prod_{j=1}^{N}\left(1-\bar{\alpha}_{j} x\right)\left(1-\bar{\alpha}_{j} y\right)\right|^{2}},
$$

$R(x, \bar{x}, y, \bar{y})$ being a polynomial of degree at most $N-2$ in each variable. For an arbitrary polynomial $f$ of degree less than $N-1$, we have the standard partial fractions decomposition

$$
\frac{f(x)}{\prod_{j=1}^{N}\left(1-\bar{\alpha}_{j} x\right)}=\sum_{j=1}^{N} \frac{A_{j}}{1-\bar{\alpha}_{j} x}
$$

for appropriate constants $A_{j}$, and $A_{j}=0$ if $\alpha_{j}=0$. Applying this procedure repeatedly to each variable, we infer that these are the partial fractions decompositions (1.32) and (1.33), and that (1.36) holds. The coefficients $e_{J K}, A_{J K L M}$ can be recaptured by taking the limits

$$
\begin{gathered}
e_{J K}(y, \eta)=\lim _{\substack{x \rightarrow 1 / \bar{\alpha}_{J} \\
\xi \rightarrow 1 / \alpha_{K}}} \frac{R(x, \xi, y, \eta) \cdot\left(1-\bar{\alpha}_{J} x\right)\left(1-\alpha_{K} \xi\right)}{\prod_{j=1}^{N}\left(1-\bar{\alpha}_{j} x\right)\left(1-\alpha_{j} \xi\right)\left(1-\bar{\alpha}_{j} y\right)\left(1-\alpha_{j} \eta\right)}, \\
A_{J K L M}=\lim _{\substack{y \rightarrow 1 / \alpha_{L} \\
\eta \rightarrow 1 / \alpha_{M}}}\left(1-\bar{\alpha}_{L} y\right)\left(1-\alpha_{M} \eta\right) \cdot e_{J K}(y, \eta) .
\end{gathered}
$$

By virtue of the well-known uniqueness theorem (cf. Bochner and Martin [1, Proposition II.4.7]), a holomorphic function $f(x, y)$ in $\mathbf{C}^{n} \times \mathbf{C}^{n}$ is uniquely determined by its restriction to the "anti-diagonal" $x=\bar{y}$. In our case, this means that we can obtain $R(x, \xi, y, \eta), e_{J K}(y, \eta)$, etc., from $R(x, \bar{x}, y, \bar{y}), e_{J K}(y, \bar{y})$, etc., by treating $x, \bar{x}$ and $y, \bar{y}$ formally as independent variables, and then replacing each occurrence of $\bar{x}$ by $\xi$ and of $\bar{y}$ by $\eta$. In particular, (1.39) is just (1.35), and (1.38) can be rewritten as (in view of (1.30), (1.31) and (1.37))

$$
e_{J K}(y, \bar{y})=\lim _{x \rightarrow 1 / \bar{\alpha}_{J}, \xi \rightarrow 1 / \alpha_{K}} \frac{\left(1-\bar{\alpha}_{J} x\right)\left(1-\alpha_{K} \xi\right) W(x, \xi)}{(1-x \xi)^{2}\left(1-|y|^{2}\right)^{2}}
$$

where

$$
W(x, \xi):=-\left(1-|y|^{2}\right)(1-x \xi) \frac{\mathcal{P}(x) \overline{\mathcal{P}(\bar{\xi})}}{(x-y)(\xi-\bar{y})}-\mathcal{P}(x) \overline{H(\bar{\xi})}-\overline{\mathcal{P}(\bar{\xi})} H(x)+h(x)+\overline{h(\bar{\xi})}
$$


Let us evaluate this limit. Owing to (1.36), we can assume that $\alpha_{J} \alpha_{K} \neq 0$. From (1.18) we see that

$$
\begin{gathered}
\lim _{x \rightarrow 1 / \bar{\alpha}_{J}}\left(1-\bar{\alpha}_{J} x\right) \frac{\mathcal{P}(x)}{x-y}=\frac{a_{J}}{1-\bar{\alpha}_{J} y}, \\
\lim _{\xi \rightarrow 1 / \alpha_{K}}\left(1-\alpha_{K} \xi\right) \frac{\overline{\mathcal{P}(\bar{\xi})}}{\xi-\bar{y}}=\lim _{\xi \rightarrow 1 / \alpha_{K}}\left(1-\alpha_{K} \xi\right) \sum_{j=1}^{N} \frac{\bar{a}_{j}}{\left(1-\alpha_{j} \xi\right)\left(1-\alpha_{j} \bar{y}\right)}=\frac{\bar{a}_{K}}{1-\alpha_{K} \bar{y}},
\end{gathered}
$$

and similarly from (1.19)

$$
\lim _{x \rightarrow 1 / \bar{\alpha}_{J}}\left(1-\bar{\alpha}_{J} x\right) H(x)=\frac{s_{J}}{\bar{\alpha}_{J}}
$$

and similarly for $\overline{H(\bar{\xi})}$, and also clearly

$$
\lim _{x \rightarrow 1 / \bar{\alpha}_{J}, \xi \rightarrow 1 / \alpha_{K}}\left(1-\bar{\alpha}_{J} x\right)\left(1-\alpha_{K} \xi\right) h(x)=0,
$$

since $h(x)$ does not depend on $\xi$; similarly for $\overline{h(\bar{\xi})}$. Putting these pieces together, (1.34) follows.

2. The case of Blaschke products. We now investigate the Green function of $\Delta\left|P^{\prime}\right|^{-2} \Delta$ for $P$ a Blaschke product. Observe that, as we have already remarked in the Introduction, if $\phi$ is a conformal map of $\mathbf{D}$ onto a Jordan domain $\Omega$, then the ordinary biharmonic operator $\Delta^{2}$ on $\Omega$ is transformed by $\phi$ into the weighted biharmonic operator $\Delta\left|\phi^{\prime}\right|^{-2} \Delta$ on $\mathbf{D}$ (cf. Proposition 2.6 below). For $\phi$ an arbitrary holomorphic function on $\mathbf{D}$, the Green function for $\Delta\left|\phi^{\prime}\right|^{-2} \Delta$ on $\mathbf{D}$ can thus be interpreted as the pullback of the ordinary (unweighted) biharmonic Green function on the Reimann surface $\Omega=\phi(\mathbf{D})$. For $\phi$ a Blaschke product, in particular, we are thus dealing with the Green function for $\Delta^{2}$ on a certain Riemann surface $\Omega$ sheeted over the unit disc $\mathbf{D}$ (with branch points at the zeros of $\phi^{\prime}$ ).

Let $B$ be a finite Blaschke product. The following lemma is very probably not new but its brief proof is included here for convenience.

Lemma 2.1. If $B$ is a Blaschke product of degree $n$, then $B^{\prime}$ has precisely $n-1$ zeros in $\mathbf{D}$, counted according to multiplicities.

Proof. Let $\alpha_{1}, \ldots, \alpha_{m}$ be the distinct zeros of $B$ and $k_{1}, \ldots, k_{m}$ their respective multiplicities. Since for Blaschke products $\overline{B(1 / \bar{x})}=B(x)$ for any $x$, the function $\sigma(x):=x B^{\prime}(x) / B(x)$ enjoys the symmetry property

$$
\sigma(x)=\overline{\sigma(1 / \bar{x})} \quad \forall x \in \mathbf{G} .
$$

If $B(0) \neq 0$, and

$$
\sigma(x)=\sum_{j=1}^{m} k_{j} \frac{\left(1-\left|\alpha_{j}\right|^{2}\right) x}{\left(x-\alpha_{j}\right)\left(1-\bar{\alpha}_{j} x\right)}
$$


then $\sigma$ is a rational function of order $2 m$, with simple poles at $\alpha_{j}$ and $1 / \bar{\alpha}_{j},(j=1, \ldots, m)$. Thus $\sigma$ has $2 m$ zeros in $\mathbf{G}$; as $\sigma(x)=\sum_{j=1}^{m} k_{j}\left(1-\left|\alpha_{j}\right|^{2}\right) /$ $\left|1-\bar{\alpha}_{j} x\right|^{2}>0$ on the unit circle, it follows from (2.1) that there must be $m$ zeros in $\Omega:=\mathbf{D} \backslash\left\{\alpha_{1}, \ldots, \alpha_{m}\right\}$ and the other $m$ in $\mathbf{G} \backslash \overline{\mathbf{D}}$. Since on $\Omega$ the zeros of $z B^{\prime}$ and of $\sigma$ obviously coincide, we see that $z B^{\prime}$ has $m$ zeros in $\Omega$ and so $B^{\prime}$ has $m-1$ zeros there. Together with the zeros of multiplicity $k_{j}-1$ at each $\alpha_{j}$, this gives $(m-1)+(n-m)=n-1$ zeros of $B^{\prime}$ in $\mathbf{D}$.

If $B(0)=0$, then say, $\alpha_{1}=0$, and (2.2) shows that $\sigma$ is of order $2 m-2$, with simple poles at $\alpha_{j}$ and $1 / \bar{\alpha}_{j}$ for $j \neq 1$. Thus it has $2 m-2$ zeros in $\mathbf{G}$ of which, by (2.2) again, $m-1$ lie in $\Omega:=\mathbf{D} \backslash\left\{0, \alpha_{2}, \ldots, \alpha_{m}\right\}$ and the other $m-1$ in $\mathbf{G} \backslash \overline{\mathbf{D}}$. As before this implies that $z B^{\prime}$ has $m-1$ zeros in $\Omega$, so that $B^{\prime}$ has $m-1$ zeros in $\Omega$ as well and together with the zeros at $0, \alpha_{2}, \ldots, \alpha_{m}$, this again yields a total of $(n-m)+(m-1)=n-1$ zeros of $B^{\prime}$ in $\mathbf{D}$.

The same argument also shows that $B^{\prime}$ has $m-1$ zeros in $\mathbf{G} \backslash \mathbf{D}$ (with at least a double zero at infinity) if $B(0) \neq 0$, and $m$ zeros there (with no zero at the infinity) if $B(0)=0$.

Corollary 2.2 Let $P$ be a Blaschke product of length $n$, with distinct zeros $\alpha_{1}, \ldots, \alpha_{m}$ of multiplicities $k_{1}, \ldots, k_{m}\left(\sum_{j=1}^{m} k_{j}=n\right)$, respectively and $\beta_{1}, \ldots, \beta_{q}$ the distinct zeros of $P^{\prime}$ in $\mathbf{D} \backslash\left\{\alpha_{1}, \ldots, \alpha_{m}\right\}$ with multiplicities $l_{1}, \ldots, l_{q}\left(\sum_{j=1}^{q} l_{j}=m-1\right)$. Then the Green function of the operator $\Delta\left|P^{\prime}\right|^{-2} \Delta$ on $\mathbf{D}$ is given by

$$
u(z, y)=\left|\frac{P(z)-P(y)}{z-y}\right|^{2} U(z, y)+\frac{\left(1-|z|^{2}\right)^{2}\left(1-|y|^{2}\right)^{2} R(z, \bar{z}, y, \bar{y})}{\left|\prod_{j=1}^{m}\left(1-\bar{\alpha}_{j} z\right)^{k_{j}}\left(1-\bar{\alpha}_{j} y\right)^{k_{j}} \cdot \prod_{j=1}^{q}\left(1-\bar{\beta}_{j} z\right)^{l_{j}}\left(1-\bar{\beta}_{j} y\right)^{l_{j}}\right|^{2}}
$$

with $R(z, \bar{z}, y, \bar{y})$ a real-valued polynomial, symmetric in $z$ and $y$, of degree not exceeding $n+m-3$ in each variable $(R \equiv 0$ if $n+m<3)$.

Proof. In the notation of the preceding section $\kappa\left(\alpha_{j}\right)=k_{j}(j=1, \ldots, m)$, $\kappa\left(\beta_{j}\right)=l_{j}(j=1, \ldots, q)$, and $\kappa(\gamma)=0$ for all other $\gamma$ in D. Thus $N=n+m-1$, and an application of Proposition 1.4 completes the proof. equal to

Corollary 2.3. For $P(z)=\frac{z-\alpha}{1-\bar{\alpha} z},(\alpha \in \mathbf{D})$, the Green function of $\Delta\left|P^{\prime}\right|^{-2} \Delta$ is

$$
u(z, y)=\left|\frac{P(z)-P(y)}{z-y}\right|^{2} U(z, y)
$$

In particular $u(z, y) \geq 0, \forall y, z \in \mathbf{D}$.

Proof. Indeed, in this case $n=m=1$, so that $R(z, \bar{z}, y, \bar{y}) \equiv 0$.

The positivity of $u$ in the last case has been established by a different method in [4]; in fact it is a simple consequence of the positivity of $U(z, y)$ and the transformation formula for the weighted biharmonic operators under a conformal mapping mentioned at the beginning of this section. Similarly, for $P$ a Blaschke product with a single zero of multiplicity $n$ at $\alpha \in \mathbf{D}$ the corresponding polynomial $R$ has degree at 
most $n-2$; for $\alpha=0$, a formula for $u$ in this case has been derived in [9], while the positivity (for any $\alpha$ ) was also proved in [4]. The first nontrivial case is thus that of a Blaschke product $P$ with two different single zeros in $\mathbf{D}$.

Let us first consider two zeros symmetric with respect to the origin.

TheOrem 2.4. Let $P=\left(z^{2}-A^{2}\right) /\left(1-\bar{A}^{2} z^{2}\right)$ be a Blaschke product with zeros $\pm A, A \in \mathbf{D} \backslash\{0\}$. Then the Green function of $\Delta\left|P^{\prime}\right|^{-2} \Delta$ is given by

$$
\begin{aligned}
u(z, y)= & \left|\frac{P(z)-P(y)}{z-y}\right|^{2} U(z, y) \\
& +\frac{\left(1-|z|^{2}\right)^{2}\left(1-|y|^{2}\right)^{2}}{\left|\left(1-\bar{A}^{2} z^{2}\right)\left(1-\bar{A}^{2} y^{2}\right)\right|^{2}} \cdot \frac{\left(1-|A|^{4}\right)^{2}}{2\left(4-|A|^{4}\right)} \operatorname{Re}\left[4-|A|^{4}+8 \bar{A}^{2} y z+4|A|^{4} y \bar{z}\right] .
\end{aligned}
$$

Proof. In this situation we have

$$
p \equiv P^{\prime}=2 z \frac{1-|A|^{4}}{\left(1-\bar{A}^{2} z^{2}\right)^{2}}, \mathcal{P} \equiv P(z)-P(y)=\frac{\left(z^{2}-y^{2}\right)\left(1-|A|^{4}\right)}{\left(1-\bar{A}^{2} z^{2}\right)\left(1-\bar{A}^{2} y^{2}\right)},
$$

and so we are in a position to apply Proposition 1.5, with $N=3, \alpha_{1}=0, \alpha_{2}=A$, $\alpha_{3}=-A$, to obtain

$$
a_{1}=0, \quad a_{2}=\left(1-|A|^{4}\right) /(2 \bar{A})=1 / \overline{p\left(\alpha_{2}\right)}, \quad a_{3}=1 / \overline{p\left(\alpha_{3}\right)}=-a_{2} .
$$

The Grammian (1.23) satisfies $G_{22}=G_{33}=\left(1-|A|^{2}\right)^{-2}, G_{23}=G_{32}=\left(1+|A|^{2}\right)^{-2}$, $G_{j 1}=G_{1 j}=1$, so that the system (1.22), (1.24) takes the simple form

$$
\begin{gathered}
s_{1}+s_{2}+s_{3}=R_{1}, \\
\bar{s}_{2} / \bar{a}_{2}+\bar{a}_{2}\left[s_{1}+G_{22} s_{2}+G_{23} s_{3}\right]=R_{2}, \\
\bar{s}_{3} / \bar{a}_{3}+\bar{a}_{3}\left[s_{1}+G_{32} s_{2}+G_{33} s_{3}\right]=R_{3},
\end{gathered}
$$

with $R_{1}, R_{2}, R_{3}$ given by (1.21). Subtracting appropriate multiples of the first equation from the other two yields

$$
\begin{gathered}
s_{1}+s_{2}+s_{3}=k, \\
\frac{\bar{s}_{2}}{\bar{a}_{2}}+\frac{1}{4}\left[\left(2-|A|^{2}\right)\left(1+|A|^{2}\right)^{2} \frac{s_{2}}{a_{2}}+\left(2+|A|^{2}\right)\left(1-|A|^{2}\right)^{2} \frac{s_{3}}{a_{3}}\right]=k_{1}, \\
\frac{\bar{s}_{3}}{\bar{a}_{3}}+\frac{1}{4}\left[\left(2+|A|^{2}\right)\left(1-|A|^{2}\right)^{2} \frac{s_{2}}{a_{2}}+\left(2-|A|^{2}\right)\left(1+|A|^{2}\right)^{2} \frac{s_{3}}{a_{3}}\right]=k_{2},
\end{gathered}
$$

where

$$
\begin{gathered}
k:=R_{1}=\frac{y\left(1-|y|^{2}\right)\left(1-|A|^{4}\right)}{1-\bar{A}^{2} y^{2}}, \\
k_{1}:=R_{2}-\bar{a}_{2} R_{1}=\frac{\left(1-|y|^{2}\right)\left(1-|A|^{4}\right)\left(1+|y|^{2}+|A|^{2} \bar{A} y-|A|^{4}|y|^{2}\right)}{2\left(1-\bar{A}^{2} y^{2}\right)(1-\bar{y} A)},
\end{gathered}
$$




$$
k_{2}:=R_{3}-\bar{a}_{3} R_{1}=\frac{\left(1-|y|^{2}\right)\left(1-|A|^{4}\right)\left(1+|y|^{2}-|A|^{2} \bar{A} y-|A|^{4}|y|^{2}\right)}{2\left(1-\bar{A}^{2} y^{2}\right)(1+\bar{y} A)} .
$$

Denoting $S:=\frac{s_{2}}{a_{2}}+\frac{s_{3}}{a_{3}}$ and $R:=\frac{s_{2}}{a_{2}}-\frac{s_{3}}{a_{3}}$, it follows that

$$
\begin{gathered}
\bar{S}=\left(k_{1}+k_{2}\right)-S, \\
\bar{R}=\left(k_{1}-k_{2}\right)-\frac{1}{2}|A|^{2}\left(3-|A|^{4}\right) R .
\end{gathered}
$$

By Proposition 1.5, the Green function for the operator $\Delta\left|P^{\prime}\right|^{-2} \Delta$ is of the form (1.31), where

$$
c(z, y)=\frac{e_{22}}{|1-\bar{A} z|^{2}}+\frac{e_{33}}{|1+\bar{A} z|^{2}}+\frac{e_{23}}{(1-\bar{A} z)(1+A \bar{z})}+\frac{e_{32}}{(1+\bar{A} z)(1-A \bar{z})},
$$

with $e_{j k}$ given by (1.34). Let us evaluate $e_{22}$, for instance. We have

$$
\bar{a}_{2} s_{2}+a_{2} \bar{s}_{2}=\left|a_{2}\right|^{2}\left(\frac{s_{2}}{a_{2}}+\frac{\bar{s}_{2}}{\bar{a}_{2}}\right)=\left|a_{2}\right|^{2} \frac{R+\bar{R}+S+\bar{S}}{2} .
$$

Adding up the equation (2.6) and its conjugate shows that

$$
\begin{aligned}
& \frac{\left(2-|A|^{2}\right)\left(1+|A|^{2}\right)^{2}}{2}(R+\bar{R}) \\
& \quad=\frac{\left(1-|y|^{2}\right)\left(1-|A|^{4}\right)}{\left|1-\bar{A}^{2} y^{2}\right|^{2}}\left(1+|A|^{2}+|y|^{2}-|A|^{4}|y|^{2}\right)(\bar{y} A+\bar{A} y),
\end{aligned}
$$

and using (2.5) it transpires that

$$
R+\bar{R}+S+\bar{S}=\frac{\left(1-|y|^{4}\right)\left(1-|A|^{4}\right)}{\left|1-\bar{A}^{2} y^{2}\right|^{2}}\left[\frac{2\left(1+|A|^{2}+|y|^{2}-|A|^{4}|y|^{2}\right)(\bar{y} A+\bar{A} y)}{\left(2-|A|^{2}\right)\left(1+|A|^{2}\right)^{2}\left(1+|y|^{2}\right)}+1\right] .
$$

Inserting this expression into (1.34) and (1.35), and using (2.4), we arrive after some simplifications at the formula

$$
e_{22}=\frac{\left(1-|A|^{4}\right)^{2}}{8\left(2-|A|^{2}\right)} \cdot \frac{2+2 \bar{A} y+2 \bar{y} A-|A|^{2}}{\left|1-\bar{A}^{2} y^{2}\right|^{2}} .
$$

In a completely similar manner it can be shown that

$$
e_{33}=\frac{\left(1-|A|^{4}\right)^{2}}{8\left(2-|A|^{2}\right)} \cdot \frac{2-2 \bar{A} y-2 \bar{y} A-|A|^{2}}{\left|1-\bar{A}^{2} y^{2}\right|^{2}}
$$

and

$$
e_{23}=\bar{e}_{32}=\frac{\left(1-|A|^{4}\right)^{2}}{8\left(2+|A|^{2}\right)} \cdot \frac{2+2 \bar{A} y-2 \bar{y} A+|A|^{2}}{\left|1-\bar{A}^{2} y^{2}\right|^{2}} \text {. }
$$


(We omit the details.) Substituting these three formulae back into (2.7), we finally obtain after a short calculation

$$
c(z, y) \cdot\left|1-\bar{A}^{2} z^{2}\right|^{2} \cdot\left|1-\bar{A}^{2} y^{2}\right|^{2} \cdot \frac{8\left(4-|A|^{4}\right)}{\left(1-|A|^{4}\right)^{2}}=4 \operatorname{Re}\left[4-|A|^{4}+8 \bar{A}^{2} y z+4|A|^{4} y \bar{z}\right],
$$

and the assertion of the theorem follows.

COROLlary 2.5 In the situation of Theorem 2.4, the Green function $u$ is positive on $\mathbf{D} \times \mathbf{D}$ if and only if $|A|^{2} \leq 2 / 5$.

Proof. For $|A| \leq \sqrt{2 / 5}$ and any $z, y \in \mathbf{D}$, we have

$$
\operatorname{Re}\left(4-|A|^{4}+8 \bar{A}^{2} y z+4|A|^{4} y \bar{z}\right)>4-|A|^{4}-8|A|^{2}-4|A|^{4}=\left(|A|^{2}+2\right)\left(2-5|A|^{2}\right) \geq 0
$$

and the positivity of $u$ follows from the well known fact that $U(z, y)>0$ on $\mathbf{D} \times \mathbf{D}$.

For the converse, let us take $y=-z \in \mathbf{D}$. Since $P$ is an even function, the first term on the RHS of (2.3) then disappears, and we get

$$
u(z,-z)=\frac{\left(1-|z|^{2}\right)^{4}}{\left|1-\bar{A}^{2} z^{2}\right|^{4}} \frac{\left(1-|A|^{4}\right)^{2}}{2\left(4-|A|^{4}\right)} \operatorname{Re}\left[4-|A|^{4}-8 \bar{A}^{2} z^{2}-4|A|^{4}|z|^{2}\right] .
$$

As $z \rightarrow A /|A| \in \partial \mathbf{D}$, the expression in the square brackets tends to

$$
4-5|A|^{4}-8|A|^{2}=\left(|A|^{2}+2\right)\left(2-5|A|^{2}\right)
$$

which is negative if $|A|^{2}>2 / 5$. Thus for $|A|>\sqrt{2 / 5}, u(z, y)$ assumes negative values in the vicinity of the point $(A /|A|,-A /|A|)$ in $\mathbf{D} \times \mathbf{D}$.

A similar argument as in the proof of Theorem 2.4 can be applied also in the case of $A=0$ (i.e. a double zero at the origin, $P(z)=z^{2}$ ) which we have excluded so far. The result is

$$
u(z, y)=|z+y|^{2} U(z, y)+\frac{1}{2}\left(1-|z|^{2}\right)^{2}\left(1-|y|^{2}\right)^{2},
$$

which, of course, agrees with the formula in [9] and also with the formula (2.3) if we set $A=0$; thus Theorem 2.4 and Corollary 2.5 remain valid for $A=0$ too.

A simple conformal invariance argument frees us from the assumption that the zeros be symmetric with respect to the origin and leads to the main result of this section.

Proposition 2.6. Let $\phi$ be a univalent holomorphic function on $\overline{\mathbf{D}}$ and $P$ a holomorphic function on $\phi(\overline{\mathbf{D}})$. Then the Green functions of the operators $\Delta\left|P^{\prime}\right|^{-2} \Delta$ on $\phi(\mathbf{D})$ and $\Delta\left|(P \circ \phi)^{\prime}\right|^{-2} \Delta$ on $\mathbf{D}$ are related by

$$
u_{P \circ \phi}(x, y)=u_{P}(\phi(x), \phi(y))
$$


In particular, if $\phi$ is a linear fractional mapping of $\mathbf{D}$ onto itself, $P$ a Blaschke product with zeros $\alpha_{1}, \alpha_{2}, \ldots$, and $P_{*}$ a Blaschke product with zeros $\phi^{-1}\left(\alpha_{1}\right), \phi^{-1}\left(\alpha_{2}\right), \ldots$ (counting multiplicities), then the Green functions $u_{P}$ and $u_{P_{*}}$ are related by $u_{P_{*}}(x, y)=U_{P}(\phi(x), \phi(y))$.

Proof. Denote, temporarily, $X:=\phi(x)$ and $Y:=\phi(y)$. Since $(P \circ \phi)^{\prime}=$ $\phi^{\prime} \cdot\left(P^{\prime} \circ \phi\right)$ and $\Delta(F \circ \phi)=\left|\phi^{\prime}\right|^{2} \cdot(\Delta F) \circ \phi$ for any function $F$, we see that

$$
\begin{aligned}
\left|(P \circ \phi)^{\prime}\right|^{-2} \Delta_{x} u_{P}(\phi(x), \phi(y)) & =\left|P^{\prime}(X)\right|^{-2}\left|\phi^{\prime}(x)\right|^{-2} \cdot\left|\phi^{\prime}(x)\right|^{2} \Delta_{X} u_{P}(X, Y) \\
& =\left(\log \left|\frac{X-Y}{1-\bar{Y} X}\right|^{2}+2 \operatorname{Re} h_{0, P}(X)\right),
\end{aligned}
$$

whence

$$
\begin{aligned}
\Delta_{x}\left|(P \circ \phi)^{\prime}\right|^{-2} \Delta_{x} u_{P}(\phi(x), \phi(y)) & =\Delta_{x} \log \left|\frac{X-Y}{1-\bar{Y} X}\right|^{2} \\
& =\left|\phi^{\prime}(x)\right|^{2} \cdot \Delta_{X} \log \left|\frac{X-Y}{1-\bar{Y} X}\right|^{2} \\
& =\left|\phi^{\prime}(x)\right|^{2} \delta_{Y}(X)=\delta_{y}(x),
\end{aligned}
$$

where $\delta$ stands for the delta function. Since $u_{P}(\phi(x), \phi(y))$ together with its first partial derivatives vanish on the boundary, it follows that $u_{P}(\phi(x), \phi(y))$ is indeed the Green function for $\Delta\left|(P \circ \phi)^{\prime}\right|^{-2} \Delta$.

For the second assertion, just observe that $P \circ \phi$ is a Blaschke product whose zeros coincide with those of $P_{*}$ (including multiplicities); hence, $P \circ \phi=\varepsilon P_{*}$, for some unimodular constant $\varepsilon$, and the claim follows.

Theorem 2.7. Let $P$ be a Blaschke product of length two with zeros $\alpha_{1}, \alpha_{2} \in \mathbf{D}$. Then the Green function of the operator $\Delta\left|P^{\prime}\right|^{-2} \Delta$ is positive if and only if $\left|\frac{\alpha_{1}-\alpha_{2}}{1-\bar{\alpha}_{1} \alpha_{2}}\right| \leq$ $\frac{2}{7} \sqrt{10}=0.9035 \ldots$

Proof. Choose a Möbius map $\phi$ (a linear fractional mapping of $\mathbf{D}$ onto itself) that carries $\alpha_{1}, \alpha_{2}$ into a pair of points symmetric with respect to the origin, $A$ and $-A$, say. By the preceding proposition and Corollary 2.5 , the Green function of $\Delta\left|P^{\prime}\right|^{-2} \Delta$ is positive if and only if $|A|^{2} \leq 2 / 5$. Since Möbius maps preserve the hyperbolic distance, we have

$$
\left|\frac{\alpha_{1}-\alpha_{2}}{1-\bar{\alpha}_{1} \alpha_{2}}\right|=\left|\frac{A-(-A)}{1-\bar{A}(-A)}\right|=\frac{2|A|}{1+|A|^{2}} .
$$

Note that the last expression is an increasing function of $|A|$ on $[0,1]$. Thus $|A| \leq \sqrt{2 / 5}$ if and only if

$$
\left|\frac{\alpha_{1}-\alpha_{2}}{1-\bar{\alpha}_{1} \alpha_{2}}\right| \leq \frac{2 \sqrt{2 / 5}}{1+\frac{2}{5}}=\frac{2}{7} \sqrt{10},
$$

and the proof is complete. 
3. The case of canonical divisors. Let $\alpha_{1}, \alpha_{2}, \ldots, \alpha_{n}$ be mutually distinct points in $\mathbf{D}, A^{2}(\mathbf{D})$ the Bergman space of square-integrable analytic functions on $\mathbf{D}$, $\mathcal{N}=\left\{f \in A^{2}(\mathbf{D}): f\left(\alpha_{j}\right)=0, \quad \forall j\right\}$ the subspace of functions that vanish at $\alpha_{1}, \ldots, \alpha_{n}$, $\beta$ a point in $\mathbf{D} \backslash\left\{\alpha_{1}, \ldots, \alpha_{n}\right\}, p$ a nonzero multiple (say $a_{0}$ ) of the orthogonal projection in $A^{2}(\mathbf{D})$ of the function $(1-\bar{\beta} z)^{-2}$ into $\mathcal{N}$, and $P$ the primitive of $p$ normalized so that $P(0)=0$.

Recall that the function $g_{x}(z):=(1-\bar{x} z)^{-2}$ is the reproducing kernel of $A^{2}(\mathbf{D})$; i.e. $\left\langle f, g_{x}\right\rangle=f(x), \forall f \in A^{2}(\mathbf{D})$. By elementary Hilbert space geometry it therefore follows that if $a_{0}$ is chosen so that $\|p\|=1$ and $p(\beta)>0, p$ will be precisely the function $G_{\alpha ; \beta}$ which maximises the value $\operatorname{Re} f(\beta)$ amongst all functions $f \in \mathcal{N}$ of norm not exceeding one:

$$
G_{\boldsymbol{\alpha} ; \beta} \in \mathcal{N},\left\|G_{\boldsymbol{\alpha} ; \beta}\right\|=1, G_{\boldsymbol{\alpha} ; \beta}(\beta)=\max \{\operatorname{Re} f(\beta): f \in \mathcal{N},\|f\| \leq 1\} .
$$

In particular, for $\beta=0, G_{\alpha ; 0}$ is Hedenmalm's canonical zero divisor $G_{\alpha}$ for the zero set $\boldsymbol{\alpha}=\left\{\alpha_{1}, \ldots, \alpha_{n}\right\}$ as discussed in the Introduction.

Since

$$
\mathcal{N}^{\perp}=\operatorname{span}\left\{\left(1-\bar{\alpha}_{j} z\right)^{-2}, j=1,2, \ldots, n\right\},
$$

we have

$$
\begin{gathered}
p(z)=\sum_{j=0}^{n} \frac{a_{j}}{\left(1-\bar{\alpha}_{j} z\right)^{2}}, \\
P(z)=\sum_{j=0}^{n} \frac{a_{j} z}{1-\bar{\alpha}_{j} z},
\end{gathered}
$$

for some $a_{1}, \ldots, a_{n}$, where $a_{0} \neq 0$, by hypothesis, and where we have set $\alpha_{0}:=\beta$. The coefficients $a_{j}$ satisfy

$$
\begin{aligned}
\sum_{j=0}^{n} a_{j}\left(1-\bar{\alpha}_{j} \alpha_{k}\right)^{-2} & =p\left(\alpha_{k}\right)=0 \quad(k=1,2, \ldots, n), \\
\sum_{j=0}^{n} a_{j}\left(1-\bar{\alpha}_{j} \beta\right)^{-2} & =p(\beta) .
\end{aligned}
$$

Also

$$
p(\beta)=\left\langle p, g_{\beta}\right\rangle=\left\langle p, g_{\beta}+\sum_{k=1}^{n} a_{k} g_{\alpha_{k}} / a_{0}\right\rangle=\left\langle p, \frac{1}{a_{0}} p\right\rangle=\|p\|^{2} / \bar{a}_{0} .
$$

In other words if we denote by $G$ the $(n+1) \times(n+1)$ matrix

$$
G=\left\{G_{i j}\right\}_{i, j=0}^{n}, \quad G_{i j}=\left(1-\bar{\alpha}_{j} \alpha_{i}\right)^{-2},
$$

and let $\left\{A_{i j}\right\}_{i, j=0}^{n}$ be its inverse, then

$$
a_{j}=\frac{\|p\|^{2}}{\bar{a}_{0}} A_{j 0} .
$$


In particular, $\left|a_{0}\right|^{2}=\|p\|^{2} A_{00}$, so that we have

$$
\|p\|^{2}=\frac{\left|a_{0}\right|^{2}}{A_{00}}, \quad p(\beta)=\frac{a_{0}}{A_{00}}, \quad a_{j}=\frac{a_{0}}{A_{00}} A_{j 0} .
$$

It is part of Hedenmalm's theory [8] that $p$ has only simple zeros at $\alpha_{1}, \ldots, \alpha_{n}$ and no other zeros in $\overline{\mathbf{D}}$. Incidentally, this also implies that all $a_{j}$ are nonzero; if, for instance, $a_{n}=0$, then $G_{\alpha_{1}, \ldots, \alpha_{n-1} ; \beta}=G_{\alpha_{1}, \ldots, \alpha_{n} ; \beta}$ would have an extra zero at $\alpha_{n}$. We are thus in a position to apply Proposition 1.5. The equations (1.22) and (1.24) take the form

$$
\begin{gathered}
\sum_{j=0}^{n} s_{j} G_{k j}=R_{k} \quad(k=1,2, \ldots, n), \\
\bar{a}_{0}^{-1} p(\beta) \bar{s}_{0}+\sum_{j=0}^{n} s_{j} G_{0 j}=R_{0},
\end{gathered}
$$

with $R_{k}$ given by (1.21). This can be solved explicitly. Adjusting $\theta$ in (1.27) if necessary we may assume that $s_{0} / a_{0} \in \mathbf{R}$. Since by (3.3) we have

$$
\sum_{j=0}^{n} a_{j} G_{k j}=0(k=1, \ldots, n), \quad \sum_{j=0}^{n} a_{j} G_{0 j}=p(\beta),
$$

we can write (3.7)-(3.8) as

$$
\sum_{j=0}^{n}\left(a_{j} s_{0}+a_{0} s_{j}\right) G_{k j}=a_{0} R_{k}, \quad k=0,1, \ldots, n .
$$

Consequently

$$
a_{m} s_{0}+a_{0} s_{m}=\sum_{k=0}^{n} a_{0} A_{m k} R_{k}(k=0,1, \ldots, n),
$$

and we thus arrive at the solution

$$
\begin{aligned}
s_{0} & =\frac{1}{2} \sum_{k=0}^{n} A_{0 k} R_{k}=\frac{a_{0}}{2\|p\|^{2}} \sum_{k=0}^{n} \bar{a}_{k} R_{k}, \\
s_{m} & =\sum_{k=0}^{n} A_{m k} R_{k}-\frac{a_{m}}{2\|p\|^{2}} \sum_{k=0}^{n} \bar{a}_{k} R_{k} .
\end{aligned}
$$

By Proposition 1.5 the sought Green function $u$ is of the form (1.31) with $c(x, y)$ given by

$$
c(x, y)=\sum_{J, K, L, M=0}^{n} \frac{A_{J K L M}}{\left(1-\bar{\alpha}_{J} x\right)\left(1-\alpha_{K} \bar{x}\right)\left(1-\bar{\alpha}_{L} y\right)\left(1-\alpha_{M} \bar{y}\right)},
$$


where the coefficients $A_{J K L M}$ can be obtained from (1.35), with $e_{J K}$ given by (1.34). Let us evaluate the limit (1.35). Owing to (1.36) we can assume that $\alpha_{L} \alpha_{M} \neq 0$. From (1.21) it then follows that

$$
\lim _{y \rightarrow 1 / \bar{\alpha}_{L}, \eta \rightarrow 1 / \alpha_{M}}\left(1-\bar{\alpha}_{L} y\right)\left(1-\alpha_{M} \eta\right) R_{k}=-\delta_{k M} \frac{a_{L}}{1-\bar{\alpha}_{L} \alpha_{M}} .
$$

Substituting this into (3.9) yields

$$
\lim _{y \rightarrow 1 / \bar{\alpha}_{L}, \eta \rightarrow 1 / \alpha_{M}}\left(1-\bar{\alpha}_{L} y\right)\left(1-\alpha_{M} \eta\right) s_{J}=-\frac{a_{L} A_{J M}}{\bar{\alpha}_{L} \alpha_{M}}+\frac{a_{J} \bar{a}_{M} a_{L}}{2\|p\|^{2} \bar{\alpha}_{L} \alpha_{M}},
$$

and similarly, upon writing $K$ in the place of $J$, interchanging $L$ and $M$, and taking complex conjugates,

$$
\lim _{y \rightarrow 1 / \alpha_{L}, \eta \rightarrow 1 / \alpha_{M}}\left(1-\bar{\alpha}_{L} y\right)\left(1-\alpha_{M} \eta\right) \overline{s_{K}}=-\frac{\bar{a}_{M} A_{L K}}{\bar{\alpha}_{L} \alpha_{M}}+\frac{\bar{a}_{K} a_{L} a_{M}}{2\|p\|^{2} \bar{\alpha}_{L} \alpha_{M}} .
$$

Also clearly

$$
\lim _{y \rightarrow 1 / \bar{\alpha}_{L}, \eta \rightarrow 1 / \alpha_{M}} \frac{\left(1-\bar{\alpha}_{L} y\right)\left(1-\alpha_{M} \eta\right)}{\left(1-\bar{\alpha}_{J} y\right)\left(1-\alpha_{K} \eta\right)}=\delta_{J L} \delta_{K M}
$$

Feeding this into (1.34) we conclude that the desired limit (1.35) satisfies

$$
\begin{aligned}
\frac{\left(1-\bar{\alpha}_{J} \alpha_{K}\right)^{2}\left(1-\bar{\alpha}_{L} \alpha_{M}\right)^{2}}{\left(\bar{\alpha}_{J} \alpha_{K} \bar{\alpha}_{L} \alpha_{M}\right)^{2}} A_{J K L M}= & -\frac{\left(1-\bar{\alpha}_{J} \alpha_{K}\right)\left(1-\bar{\alpha}_{L} \alpha_{M}\right)}{\bar{\alpha}_{J} \alpha_{K} \bar{\alpha}_{L} \alpha_{M}} a_{J} \bar{a}_{K} \delta_{J L} \delta_{K M} \\
& +\frac{\bar{a}_{M} a_{J} A_{L K}+\bar{a}_{K} a_{L} A_{J M}}{\bar{\alpha}_{J} \alpha_{K} \bar{\alpha}_{L} \alpha_{M}}-\frac{a_{J} \bar{a}_{K} a_{L} \bar{a}_{M}}{\|p\|^{2} \bar{\alpha}_{J} \alpha_{K} \bar{\alpha}_{L} \alpha_{M}} .
\end{aligned}
$$

Substituting for $a_{j}$ from (3.6) and using (3.4) we finally obtain

$$
\begin{aligned}
& A_{J K L M}=\bar{\alpha}_{J} \alpha_{K} \bar{\alpha}_{L} \alpha_{M} G_{K J} G_{M L} \cdot \frac{|p(\beta)|^{4}}{\|p\|^{2}} \times \\
& \times\left[-\frac{A_{00} A_{J 0} A_{0 K} \delta_{J L} \delta_{K M}}{G_{K J}}+A_{00} A_{J 0} A_{0 M} A_{L K}+A_{00} A_{L 0} A_{0 K} A_{J M}-A_{J 0} A_{0 K} A_{L 0} A_{0 M}\right] .
\end{aligned}
$$

Even though this formula has been derived for $\alpha_{L} \alpha_{M} \neq 0$, it gives the correct answer even if $\alpha_{L}$ or $\alpha_{M}$ vanish, since $A_{J K L M}=0$ in that case by (1.36). Thus (3.11) is valid for all $J, K, L, M$.

We have arrived at the following theorem.

THEOREM 3.1. Let $\alpha_{1}, \alpha_{2}, \ldots, \alpha_{n}$ and $\alpha_{0}=\beta$ be distinct points in $\mathbf{D}$ and $p$ a function satisfying

$$
p(z)=\sum_{j=0}^{n} a_{j}\left(1-\bar{\alpha}_{j} z\right)^{-2}, p\left(\alpha_{k}\right)=0(k=1,2, \ldots, n), p(\beta) \neq 0 .
$$


Then the Green function for the operator $\Delta|p|^{-2} \Delta$ is equal to

$$
\begin{aligned}
u(x, y)= & \left|\frac{P(x)-P(y)}{x-y}\right|^{2} U(x, y) \\
& +\left(1-|x|^{2}\right)^{2}\left(1-|y|^{2}\right)^{2} \frac{|p(\beta)|^{4}}{\|p\|^{2}} \sum_{J, K, L, M=0}^{n} a_{J K L M} X_{J} \bar{X}_{K} Y_{L} \bar{Y}_{M},
\end{aligned}
$$

where

$$
\begin{array}{r}
a_{J K L M}=G_{K J} G_{M L}[- \\
\quad \frac{A_{00} A_{J 0} A_{0 K} \delta_{J L} \delta_{K M}}{G_{K J}}+A_{00} A_{J 0} A_{0 M} A_{L K} \\
\left.+A_{00} A_{L 0} A_{0 K} A_{J M}-A_{J 0} A_{0 K} A_{L 0} A_{0 M}\right]
\end{array}
$$

Here $P$ is a primitive to $p, U(x, y)$ is the Green function for the ordinary bilaplacian $\Delta^{2}, X_{j}:=\bar{\alpha}_{j} /\left(1-\bar{\alpha}_{j} x\right), Y_{j}:=\bar{\alpha}_{j} /\left(1-\bar{\alpha}_{j} y\right)$, and $\left\{A_{j k}\right\}_{j, k=0}^{n}$ is the inverse of the Grammian matrix $G=\left\{G_{j k}\right\}_{j, k=0}^{n}, G_{i j}:=\left(1-\bar{\alpha}_{j} \alpha_{i}\right)^{-2}$.

The following special case has been independently obtained by a different method by Hedenmalm (unpublished).

COROLlary 3.2. Let $p$ be the canonical zero-divisor of a finite zero-set $\left\{\alpha_{1}, \ldots, \alpha_{n}\right\}$ consisting of distinct points in $\mathbf{D} \backslash\{0\}$. Then the Green function for the operator $\Delta|p|^{2} \Delta$ is equal to

$$
\begin{aligned}
& u(x, y)=\left|\frac{P(x)-P(y)}{x-y}\right|^{2} U(x, y)+\left(1-|x|^{2}\right)^{2}\left(1-|y|^{2}\right)^{2} p(0)^{-2} \times \\
& \times \sum_{J, K, L, M=1}^{n} X_{J} \bar{X}_{K} Y_{L} \bar{Y}_{M} G_{K L} G_{M L} \cdot \mu_{J} \bar{\mu}_{K} \mu_{L} \bar{\mu}_{M}\left[-\frac{\delta_{J L} \delta_{K M}}{G_{K J} \mu_{J} \bar{\mu}_{K}}+\frac{\mu_{L K}}{\mu_{L} \bar{\mu}_{K}}+\frac{\mu_{J M}}{\mu_{J} \bar{\mu}_{M}}+\frac{1}{p(0)^{2}}\right] .
\end{aligned}
$$

Here $P$ is a primitive to $p, U(x, y)$ is the Green function for the ordinary bilaplacian $\Delta^{2}, X_{j}:=\bar{\alpha}_{j} /\left(1-\bar{\alpha}_{j} x\right), Y_{j}:=\bar{\alpha}_{j} /\left(1-\bar{\alpha}_{j} y\right),\left\{\mu_{j k}\right\}_{j, k=1}^{n}$ is the inverse of the $n \times n$ matrix $\left\{G_{j k}\right\}_{j, k=1}^{n}, G_{j k}=\left(1-\bar{\alpha}_{k} \alpha_{j}\right)^{-2}$, and $\mu_{i}=\sum_{j=1}^{n} \mu_{i j}$.

Proof. As we have already observed at the beginning of this section, the canonical divisor $p$ is a function of the form (3.12) which in addition satisfies

$$
\|p\|=1, p(0)=\|p\|^{2} / \bar{a}_{0}>0, \beta=0 .
$$

Thus we can apply the last theorem. Since by (3.3) and (3.6) we have

$$
\begin{aligned}
\sum_{k=1}^{n}\left(A_{m k}-\frac{a_{m} \bar{a}_{k}}{\|p\|^{2}}\right) G_{k j} & =\left(\delta_{m j}-A_{m 0} G_{0 j}\right)-\frac{a_{m}}{\|p\|^{2}}\left(\sum_{k=0}^{n} \overline{G_{j k} a_{k}}-\overline{G_{j 0} a_{0}}\right) \\
& =\left(\delta_{m j}-\frac{\bar{a}_{0} a_{m}}{\|p\|^{2}} G_{0 j}\right)-\frac{a_{m}}{\|p\|^{2}}\left(\overline{p\left(\alpha_{j}\right)}-\overline{G_{j 0} a_{0}}\right) \\
& =\delta_{m j},
\end{aligned}
$$


for any $j, m=1, \ldots, n$, the coefficients $\mu_{m k}$ are connected with $A_{m k}$ by the relation

$$
A_{m k}=\mu_{m k}+a_{m} \bar{a}_{k}
$$

Further, by (3.3) $\sum_{j=1}^{n} G_{k j} a_{j}=-G_{k 0} a_{0}=-a_{0}$ for $k=1, \ldots, n$, whence

$$
a_{m}=-\sum_{k=1}^{n} \mu_{m k} a_{0}=-a_{0} \mu_{m}=-\frac{1}{p(0)} \mu_{m}
$$

Finally, as $\beta=0$, we have $X_{0}=Y_{0}=0$ and so the summation in (3.13) is effectively carried out only over $J, K, L, M$ from 1 to $n$. Inserting (3.16) and (3.17) into (3.13), we therefore obtain (3.15).

Let us record also the following invariance property of the sum in (3.13).

Proposition 3.3. For $\mathbf{x}=\left(x_{j}\right)_{j=0}^{n}$ and $\mathbf{y}=\left(y_{j}\right)_{j=0}^{n}$, two vectors in $\mathbf{C}^{n+1}$, denote

$$
\sigma_{\alpha_{1}, \ldots, \alpha_{n} ; \beta}(\mathbf{x}, \mathbf{y})=\frac{1}{A_{00}^{2}} \sum_{j, k, l, m=0}^{n} a_{j k l m} x_{j} \bar{x}_{k} y_{l} \bar{y}_{m}
$$

with $a_{j k l m}$ given by (3.14). Then for any holomorphic self-map $\phi$ of the unit disc $\mathbf{D}$

$$
\sigma_{\phi\left(\alpha_{1}\right), \ldots, \phi\left(\alpha_{n}\right) ; \phi(\beta)}(\mathbf{x}, \mathbf{y})=\sigma_{\alpha_{1}, \ldots, \alpha_{n} ; \beta}(\mathbf{x}, \mathbf{y}) .
$$

Proof. Let $\phi(z)=\epsilon(z-\alpha) /(1-\bar{\alpha} z), \alpha \in \mathbf{D},|\epsilon|=1$. A simple calculation reveals that

$$
\left(1-\overline{\phi\left(\alpha_{k}\right)} \phi\left(\alpha_{j}\right)\right)^{2}=\left[\frac{\left(1-\bar{\alpha}_{k} \alpha_{j}\right)\left(1-|\alpha|^{2}\right)}{\left(1-\bar{\alpha}_{k} \alpha\right)\left(1-\bar{\alpha} \alpha_{j}\right)}\right]^{2}=\left(1-\bar{\alpha}_{k} \alpha_{j}\right)^{2} \cdot \overline{\phi^{\prime}\left(\alpha_{k}\right)} \phi^{\prime}\left(\alpha_{j}\right)
$$

Thus upon replacing $\left\{\alpha_{j}\right\}$ by $\left\{\phi\left(\alpha_{j}\right)\right\}$, the matrix $\left(G_{j k}\right)$ changes into $\left(G_{j k} /\left(\phi^{\prime}\left(\alpha_{j}\right) \overline{\phi^{\prime}\left(\alpha_{k}\right)}\right)\right)$, so that its inverse $\left(A_{j k}\right)$ changes into $\left(\overline{\phi^{\prime}\left(\alpha_{j}\right)} \phi^{\prime}\left(\alpha_{k}\right) A_{j k}\right)$, and the assertion follows.

In terms of $\sigma$, the formula (3.13) for the Green function becomes

$$
\begin{aligned}
u(x, y) & =\left|\frac{P(x)-P(y)}{x-y}\right|^{2} U(x, y) \\
& +\left(1-|x|^{2}\right)^{2}\left(1-|y|^{2}\right)^{2}\|p\|^{2} \sigma_{\alpha_{1}, \ldots, \alpha_{n} ; \beta}\left(\left(\frac{\bar{\alpha}_{j}}{1-\bar{\alpha}_{j} x}\right),\left(\frac{\bar{\alpha}_{j}}{1-\bar{\alpha}_{j} y}\right)\right) .
\end{aligned}
$$

For $n=1$, the sum (3.18) is easily evaluated explicitly:

$$
\sigma_{\alpha ; \beta}(\mathbf{x}, \mathbf{y})=\frac{\left(1-|\alpha|^{2}\right)^{2} A_{00}}{|1-\bar{\alpha} \beta|^{4}-\left(1-|\alpha|^{2}\right)^{2}\left(1-|\beta|^{2}\right)^{2}} \cdot\left|x_{0}-x_{1}\right|^{2}\left|y_{0}-y_{1}\right|^{2} .
$$

Thus we obtain the following result.

Proposition 3.4. Consider the situation as in Theorem 3.1, with $n=1, \alpha_{1}=\alpha$, $\alpha_{0}=\beta$. Then the Green function is given by the formula 


$$
\begin{aligned}
u(z, y) & =\left|\frac{P(z)-P(y)}{z-y}\right|^{2} U(z, y) \\
& +\frac{\left(1-|\alpha|^{2}\right)^{2}\left(1-|\beta|^{2}\right)^{2}}{\left(2-\left|\frac{\alpha-\beta}{1-\bar{\alpha} \beta}\right|^{2}\right)^{2}} \cdot \frac{\|p\|^{2}\left(1-|z|^{2}\right)^{2}\left(1-|y|^{2}\right)^{2}}{|(1-\bar{\alpha} z)(1-\bar{\alpha} y)(1-\bar{\beta} z)(1-\bar{\beta} y)|^{2}} .
\end{aligned}
$$

In particular, $u(z, y)$ is positive on $\mathbf{D} \times \mathbf{D}$.

For $n>1$ the biquadratic form (3.18) becomes fairly complex and the author was unable to obtain a closed expression for it even in the simplest case $n=2$. Computer-based calculations, however, seem to give evidence in support of the following conjecture.

\section{Conjecture 3.5. Denote}

$$
\varrho_{\alpha_{1}, \ldots, \alpha_{n} ; \beta}(\xi, \eta)=\frac{1}{A_{00}^{2}} \sum_{j, k, l, m=0}^{n} a_{j k l m} \xi_{j l} \bar{\eta}_{k m}\left(\xi, \eta \in \mathbf{C}^{(n+1)^{2}}\right) .
$$

The quadratic form $\varrho_{\alpha_{1}, \ldots \alpha_{n} ; \beta}(\xi, \xi)$ in the $(n+1)^{2}$ complex variables $\left(\xi_{i j}\right)_{j=0}^{n}$ is positive semidefinite.

In particular, taking $\xi_{j l}=x_{j} y_{l}, \sigma_{\alpha_{1}, \ldots, \alpha_{n} ; \beta}(\mathbf{x}, \mathbf{y}) \geq 0$ for all $\mathbf{x}, \mathbf{y} \in \mathbf{C}^{n+1}$ and mutually distinct points $\alpha_{1}, \alpha_{2}, \ldots, \alpha_{n}, \beta \in \mathbf{D}$.

REMARK. The analogous quadratic form obtained from (3.18) upon introducing instead the variables $\eta_{j m}=x_{j} \bar{y}_{m}$ is not positive semidefinite in general. A counterexample is $n=2$ and $\alpha_{0}=0, \alpha_{1}=1 / 3, \alpha_{2}=2 / 3$; the quadratic form has then three positive, five zero and one negative eigenvalues. We also remark that the points $\alpha_{1}, \ldots, \alpha_{n}, \beta$ enter into (3.18) and (3.20) (i.e., into $a_{j k l m}$ ) only through the coefficients of the matrices $\left(G_{j k}\right)$ and $\left(A_{j k}\right)$, and thus it makes sense to define $\sigma(\mathbf{x}, \mathbf{y})$ and $\varrho(\zeta, \eta)$, more generally, for any positive definite (or even nonsingular) matrix $G$ and its inverse $A$, and question their positivity in this more general setting. Then it is possible to obtain "counterexamples" to Conjecture 3.5 as well, but neither the corresponding matrices $G$ (one of them is e.g. $G=\left(\begin{array}{ccc}1 & 1 / \sqrt{3} & 1 / \sqrt{3} \\ 1 / \sqrt{3} & 1 & 1 / 3 \\ 1 / \sqrt{3} & 1 / 3 & 1\end{array}\right)$ for $n=2$ ) nor even any of their conjugates $D^{*} G D$ by diagonal matrices $D$ are of the form (3.5) for $\alpha_{0}, \alpha_{1}, \alpha_{2} \in \mathbf{D}$. The author does not know which positive definite matrices arise as $D^{*} G D$ with $D$ diagonal and $G$ as in (3.5) - that is, in other words, what arrangements of angles can there occur between multiples of the reproducing kernels $g_{\alpha_{1}}, \ldots, g_{\alpha_{m}} \in A^{2}(\mathbf{D})$ of $m$ distinct points in D. For $m=2$, these are precisely all positive definite matrices whose off-diagonal terms are non-zero.

Owing to the following invariance property, it would be enough to verify Conjecture 3.5 for $\beta=0$.

Proposition 3.6. For any holomorphic self-map $\phi$ of the unit disc $\mathbf{D}$, we have

$$
\varrho_{\phi\left(\alpha_{1}\right), \ldots, \phi\left(\alpha_{n}\right) ; \phi(\beta)}(\xi, \eta)=\varrho_{\alpha_{1}, \ldots, \alpha_{n} ; \beta}(\xi, \eta) .
$$

Proof. This is the same as Proposition 3.3. 
We close by mentioning a curious operator-theoretic interpretation of the biquadratic form (3.18). Consider quite generally a Hilbert space $\mathcal{H}$ of dimension $n+1$ spanned by linearly independent vectors $g_{0}, g_{1}, \ldots, g_{n}$ (not necessarily unit or orthogonal) and let $\gamma_{0}, \ldots, \gamma_{n}$ be the dual basis:

$$
\left\langle g_{j}, \gamma_{k}\right\rangle= \begin{cases}1 & j=k \\ 0 & j \neq k\end{cases}
$$

The corresponding Grammians

$$
G_{j k}:=\left\langle g_{k}, g_{j}\right\rangle, \quad A_{j k}:=\left\langle\gamma_{k}, \gamma_{j}\right\rangle
$$

are then related by $G=A^{-1}$. For any $(n+1)$-tuple of complex numbers $\mathbf{x}=\left(x_{0}, \ldots, x_{n}\right)$, define the operator $\mathbf{X}$ on $\mathcal{H}$ by

$$
\mathbf{X}: \sum_{j} c_{j} \gamma_{j} \mapsto \sum_{j} \bar{x}_{j} c_{j} \gamma_{j}
$$

(that is, $\left\{\gamma_{j}\right\}$ is a complete system of eigenvectors for $\mathbf{X}$ with $\bar{x}_{j}$ the corresponding eigenvalues), and let $\mathbf{Y}$ be defined similarly for $\mathbf{y}=\left(y_{0}, \ldots, y_{n}\right)$. One easily computes that the adjoint operators are given by

$$
\mathbf{X}^{*}: \sum_{j} c_{j} \gamma_{j} \mapsto \sum_{j, k, m} x_{m} c_{j} A_{m j} G_{k m} \gamma_{k}
$$

and similarly for Y. In particular,

$$
\begin{aligned}
\left\langle\mathbf{X X}^{*} \gamma_{0}, \mathbf{Y Y}^{*} \gamma_{0}\right\rangle & =\left\langle\sum_{k, m} \bar{x}_{k} x_{m} A_{m 0} G_{k m} \gamma_{k}, \sum_{K, M} \bar{y}_{K} y_{M} A_{M 0} G_{K M} \gamma_{K}\right\rangle \\
& =\sum_{k, m, K, M} x_{m} \bar{x}_{k} y_{K} \bar{y}_{M} G_{k m} G_{M K} A_{m 0} A_{0 M} A_{K k} \\
& =\sum_{j, k, l, m} x_{j} \bar{x}_{k} y_{l} \bar{y}_{m} G_{k j} G_{m l} A_{j 0} A_{0 m} A_{l k} .
\end{aligned}
$$

In the special case $y_{j}=1, \forall j$ this gives

$$
\left\langle\mathbf{X X}^{*} \gamma_{0}, \gamma_{0}\right\rangle=\left\|\mathbf{X}^{*} \gamma_{0}\right\|^{2}=\sum_{j k} x_{j} \bar{x}_{k} G_{k l} A_{j 0} A_{0 k},
$$

and similarly for $\mathbf{Y}$. Also the $n$-tuple $\left(x_{0} y_{0}, \ldots, x_{n} y_{n}\right)$ corresponds to the operator $\mathbf{X Y}(=\mathbf{Y X})$, so that

$$
\left\|\mathbf{X}^{*} \mathbf{Y}^{*} \gamma_{0}\right\|^{2}=\sum_{j k} x_{j} \bar{x}_{k} y_{j} \bar{y}_{k} G_{k j} A_{j 0} A_{0 k}
$$

Finally note that $\left\|\gamma_{0}\right\|^{2}=A_{00}$ by definition. Putting everything together we thus see that 


$$
\begin{gathered}
\frac{\left\langle\mathbf{X} \mathbf{X}^{*} \gamma_{0}, \mathbf{Y} \mathbf{Y}^{*} \gamma_{0}\right\rangle}{\left\|\gamma_{0}\right\|^{2}}+\frac{\left\langle\mathbf{Y} \mathbf{Y}^{*} \gamma_{0}, \mathbf{X X}^{*} \gamma_{0}\right\rangle}{\left\|\gamma_{0}\right\|^{2}}-\frac{\left\|\mathbf{X}^{*} \gamma_{0}\right\|^{2}\left\|\mathbf{Y}^{*} \gamma_{0}\right\|^{2}}{\left\|\gamma_{0}\right\|^{4}}-\frac{\left\|\mathbf{X}^{*} \mathbf{Y}^{*} \gamma_{0}\right\|^{2}}{\left\|\gamma_{0}\right\|^{2}}= \\
=\frac{1}{A_{00}^{2}} \sum_{j, k, l, m=0}^{n} x_{j} \bar{x}_{k} y_{l} \bar{y}_{m} G_{k j} G_{m l}\left[A_{00} A_{j 0} A_{0 m} A_{l k}+A_{00} A_{l 0} A_{0 k} A_{j m}\right. \\
\left.\quad-A_{j 0} A_{0 k} A_{l 0} A_{0 m}-A_{00} \delta_{j l} \delta_{k m} \frac{A_{j 0} A_{0 k}}{G_{k j}}\right] .
\end{gathered}
$$

Let us now apply this to the situation in which $g_{j}=\left(1-\bar{\alpha}_{j} z\right)^{-2} \in A^{2}(\mathbf{D})$ are the reproducing kernels for some distinct points $\alpha_{0}, \ldots, \alpha_{n} \in \mathbf{D}$. The corresponding Grammian (3.22) then coincides with (3.5), $\mathcal{H}=A^{2}(\mathbf{D}) \ominus \mathcal{M}$, where $\mathcal{M}=\mathcal{N}_{\alpha \cup\{\beta\}}$ is the subspace of functions that vanish at $\alpha_{0}, \ldots, \alpha_{n}$, and the vector $\gamma_{0}$ of the dual basis (3.21) is, in view of (3.3) and (3.4), given by

$$
\gamma_{0}=\frac{\bar{a}_{0}}{\|p\|^{2}} p .
$$

Comparing (3.24) with (3.18) we get

$$
2 \operatorname{Re} \frac{\left\langle\mathbf{X X}^{*} p, \mathbf{Y} \mathbf{Y}^{*} p\right\rangle}{\|p\|^{2}}-\frac{\left\|\mathbf{X}^{*} \mathbf{Y}^{*} p\right\|^{2}}{\|p\|^{2}}-\frac{\left\|\mathbf{X}^{*} p\right\|^{2}\left\|\mathbf{Y}^{*} p\right\|^{2}}{\|p\|^{4}}=\sigma_{\alpha_{1}, \ldots, \alpha_{n} ; \beta}(\mathbf{x}, \mathbf{y}),
$$

where $\mathbf{X}$ and $\mathbf{Y}$ are operators on $\mathcal{H}$ given by (3.23). These operators can be expressed in terms of the operator $S$ of multiplication by $z$ on $A^{2}(\mathbf{D})$ (the Bergman shift):

$$
S: A^{2}(\mathbf{D}) \rightarrow A^{2}(\mathbf{D}),(S f)(z):=z f(z) .
$$

Indeed, in terms of the dual basis (3.21), (3.23) becomes

$$
\mathbf{X}^{*}: \sum_{j} c_{j} g_{j} \mapsto \sum_{j} x_{j} c_{j} g_{j}
$$

On the other hand, it is well known that $S^{*} g_{j}=\bar{\alpha}_{j} g_{j}$. (In particular, $\mathcal{H}$ is an invariant subspace for $S^{*}$.) Thus if $\phi_{x}$ is a function holomorphic in a neighbourhood of $\overline{\mathbf{D}}=$ $\operatorname{Spectrum}\left(S^{*}\right)$ such that

$$
\phi_{x}\left(\bar{\alpha}_{j}\right)=x_{j}(j=0,1, \ldots, n)
$$

then $\phi_{x}\left(S^{*}\right)=\mathbf{X}^{*}$ on $\mathcal{H}$. Similarly for $\mathbf{Y}$.

In particular, for $x_{j}=\bar{\alpha}_{j} /\left(1-\bar{\alpha}_{j} x\right)$ with some $x \in \mathbf{D}$, one can take $\phi_{x}(z)=z /(1-x z)$; using (3.19), we thus obtain the following proposition.

Proposition 3.7. Let $\alpha_{1}, \ldots, \alpha_{n}$ and $\alpha_{0}=\beta$ be mutually distinct points in $\mathbf{D}, S$ the Bergman shift (3.26) and $\mathcal{H}$ the orthogonal complement in $A^{2}(\mathbf{D})$ of the subspace of functions that vanish at $\alpha_{0}, \alpha_{1}, \ldots, \alpha_{n}$. For any $x \in \mathbf{D}$, let $S_{x}^{*}: \mathcal{H} \rightarrow \mathcal{H}$ be the restriction of the operator $S^{*}\left(1-x S^{*}\right)^{-1}$ to its invariant subspace $\mathcal{H}$, and let $S_{x}: \mathcal{H} \rightarrow \mathcal{H}$ be its adjoint. Let $S_{y}^{*}$ and $S_{y}$ be defined similarly for $y \in \mathbf{D}$. Then the Green function (3.13) from Theorem 3.1 is equal to 


$$
u(x, y)=\left|\frac{P(x)-P(y)}{x-y}\right|^{2} U(x, y)+\left(1-|x|^{2}\right)^{2}\left(1-|y|^{2}\right)^{2}\|p\|^{2} c(x, y),
$$

where

$$
c(x, y)=-\frac{\left\|S_{x}^{*} S_{y}^{*} p\right\|^{2}}{\|p\|^{2}}-\frac{\left\|S_{x}^{*} p\right\|^{2}\left\|S_{y}^{*} p\right\|^{2}}{\|p\|^{4}}+2 \operatorname{Re} \frac{\left\langle S_{x} S_{x}^{*} p, S_{y} S_{y}^{*} p\right\rangle}{\|p\|^{2}} .
$$

REMARK. Explicitly, the operator $S_{x}$ is given by $S_{x}=\left.P_{\mathcal{H}} S(1-\bar{x} S)^{-1}\right|_{\mathcal{H}}$, where $P_{\mathcal{H}}$ is the orthogonal projection from $A^{2}(\mathbf{D})$ onto $\mathcal{H}$. In fact, the operators $\phi_{x}\left(S^{*}\right)$ above are just the Toeplitz operators on $A^{2}(\mathbf{D})$ with coanalytic symbols, while the $S_{x}$ are a Bergman-space analogue of the "model operators" of dilation theory on $H^{2}$ (cf. [11, Chapter VIII]).

COROllary 3.8. Let $\alpha=\left\{\alpha_{1}, \ldots, \alpha_{n}\right\}$ be a sequence of points in $\mathbf{D}$ (not necessarily distinct), $\beta$ any point in $\mathbf{D}$, and $G_{\alpha ; \beta}$ the (unique) solution of the extremal problem

$$
\max \left\{\operatorname{Re} f^{(m)}(\beta) ;\|f\| \leq 1, f \text { vanishes on } \alpha \text { (including multiplicities) }\right\},
$$

where $m$ is the multiplicity of $\beta$ in the sequence $\alpha$. Then the corresponding Green function $u_{\boldsymbol{\alpha} ; \beta}(x, y)$ of the operator $\Delta\left|G_{\boldsymbol{\alpha} ; \beta}\right|^{-2} \Delta$ on $\mathbf{D}$ (exists and) depends continuously on $\alpha_{1}, \ldots, \alpha_{n}, \beta \in \mathbf{D}$.

Proof. Set as before $\alpha_{0}:=\beta$ and consider the operators

$$
T_{\alpha ; \beta}: f(z) \mapsto f(z) \cdot \prod_{j=0}^{n}\left(z-\alpha_{j}\right) .
$$

This is a family of bounded operators on $A^{2}(\mathbf{D})$ which depends norm-continuously on $(\alpha ; \beta) \in \mathbf{D}^{n+1}$; moreover, they are bounded below; $\left\|T_{\alpha ; \beta}\right\| \geq c_{\alpha ; \beta}\|f\|$ for some $c_{\boldsymbol{\alpha} ; \beta}>0$, and uniformly so when $(\alpha ; \beta)$ ranges in a compact subset of $\mathbf{D}^{n+1}$. Both properties remain in force also for the positive operators $P_{\alpha ; \beta}=T_{\alpha ; \beta}^{*} T_{\alpha ; \beta} \geq C_{\alpha ; \beta}^{2} I$; using the holomorphic functional calculus, we infer that the $P_{\alpha ; \beta}^{-1 / 2}$ depend, likewise, continuously on $(\alpha ; \beta)$. Hence, so do the partial-isometry components $W_{\alpha ; \beta}=T_{\alpha ; \beta} P_{\alpha ; \beta}^{-1 / 2}$ in the polar decomposition of $T_{\alpha ; \beta}\left(W_{\alpha ; \beta}\right.$ is in fact an isometry of $A^{2}$ (D) onto the subspace $\mathcal{M}_{\alpha ; \beta}:=\mathcal{N}_{\alpha \cup\{\beta\}}$ of functions which vanish at $\alpha_{1}, \ldots, \alpha_{n}, \beta$ counting multiplicities) and the product $W_{\alpha ; \beta} W_{\alpha ; \beta}^{*}$, which is the orthogonal projection onto Range $\left(T_{\alpha ; \beta}\right)=\mathcal{M}_{\alpha ; \beta}$, as well as the projection $I-W_{\boldsymbol{\alpha} ; \beta} W_{\alpha ; \beta}^{*}$ onto the orthogonal complement $\mathcal{H}_{\boldsymbol{\alpha} ; \beta}=A^{2}(\mathbf{D}) \ominus \mathcal{M}_{\boldsymbol{\alpha} ; \beta}$. A similar argument applies, of course, to the operators

$$
T_{\alpha}: f(z) \mapsto f(z) \cdot \prod_{j=1}^{n}\left(z-\alpha_{j}\right)
$$

obtained upon omitting the point $\beta$, and shows that the orthogonal projection of $A^{2}(\mathbf{D})$ onto $\mathcal{N}_{\alpha}$ depends continuously on $\alpha \in \mathbf{D}^{n}$. As $\mathcal{N}_{\alpha} \supset \mathcal{M}_{\alpha ; \beta}$ and $\mathcal{N}_{\boldsymbol{\alpha}} \ominus \mathcal{M}_{\boldsymbol{\alpha} ; \beta}=\mathbf{C G}_{\boldsymbol{\alpha} ; \beta}$ is precisely the one-dimensional subspace spanned by the 
solution $G_{\alpha ; \beta}$ of the extremal problem (3.29), we see that this one-dimensional subspace, call it $\mathcal{G}_{\alpha ; \beta}$, also depends continuously on $(\alpha ; \beta)$. Thus both the operators $\left(I-W_{\alpha ; \beta} W_{\alpha ; \beta}^{*}\right) . S(1-\bar{x} S)^{-1} .\left(I-W_{\alpha ; \beta} W_{\alpha ; \beta}^{*}\right)=S_{x}$ and $S_{y}$ in (3.28) depend continuously on $(\alpha ; \beta)$, and so does the one-dimensional subspace spanned by the $p$ there; as (3.28) is evidently homogenous of degree 0 in $p$, it follows that the expression $c(x, y)$ given by (3.28) with $p=G_{\alpha ; \beta}$ extends by continuity (for each fixed $x$ and $y$ ) to a continuous function on all $(\alpha ; \beta) \in \mathbf{D}^{n+1}$ (i.e. even to the $\alpha_{0}, \alpha_{1}, \ldots, \alpha_{n}$ which are not mutually distinct). Similarly, the number

$$
\left|\frac{P(x)-P(y)}{x-y}\right|^{2}=\left|\int_{[x, y]} p(z) d z\right|^{2}
$$

is unchanged if we replace $p$ by $\epsilon p$ with a unimodular $\epsilon$, so that it yields the same value regardless of the choice of the unit vector $p$ in the one-dimensional space $\mathcal{G}_{\alpha ; \beta}$, and this value thus varies continuously with $(\alpha ; \beta)$; the same is of course true for $\|p\|^{2}(=1)$, and so we finally see that the expression $u(x, y)$ given by (3.27) with $p=G_{\boldsymbol{\alpha} ; \beta}$ defines, for each fixed $x$ and $y$, a continuous function on all $\mathbf{D}^{n+1}$. However, both (3.30) and $c(x, y)$ are rational functions in $\alpha_{0}, \alpha_{1}, \ldots, \alpha_{n}$ (since $G_{j k}$, $\operatorname{det}\left(G_{j k}\right)$ and, hence, $A_{j k}$ are); thus the identity

$$
\Delta_{x}\left[\left|G_{\boldsymbol{\alpha} ; \beta}(x)\right|^{-2} \Delta_{x} u_{\boldsymbol{\alpha} ; \beta}(x, y)-\Gamma(x, y)\right]=0 \quad \forall x, y \in \mathbf{D},
$$

which we know to hold true when $\alpha_{0}, \alpha_{1}, \ldots, \alpha_{n}$ are mutually distinct by the last proposition, must in fact be valid universally.

REMARK. One more virtue of the formula (3.25) is that it shows immediately that $\sigma(\mathbf{x}, \mathbf{y})=0$ if $\mathbf{x}$ or $\mathbf{y}$ is a scalar multiple of $(1,1,1, \ldots, 1)$. In terms of the quadratic form (3.20) this means that

$$
\varrho(\xi, \eta)=0, \forall \eta \quad \text { if } \xi=r_{I} \text { or } \xi=c_{J},
$$

where $\left(r_{I}\right)_{i j}:=\delta_{i I}$ and $\left(c_{J}\right)_{i j}:=\delta_{j J}$. Thus the subspace $\mathcal{N}_{0} \subset \mathbf{C}^{(n+1)^{2}}$ spanned by $r_{I}$ and $c_{I}, I=0,1, \ldots, n$ (its dimension is $2 n+1$, since $\sum_{I} r_{I}=\sum_{J} c_{J}$ ) is contained in the kernel of the quadratic form $\varrho(\xi, \xi)$. Moreover, the two subspaces

$$
\mathcal{N}_{+}:=\left\{\xi \in \mathcal{N}_{0}^{\perp}: \xi_{i j}=\xi_{j i}\right\}, \quad \mathcal{N}_{-}:=\left\{\xi \in \mathcal{N}_{0}^{\perp}: \xi_{i j}=-\xi_{j i}\right\}
$$

are easily seen to be orthogonal under $\varrho$, a reflection of the fact that $\sigma(\mathbf{x}, \mathbf{y})$ is symmetric in $\mathbf{x}$ and $\mathbf{y}$. Thus if $\xi=\xi_{0}+\xi_{+}+\xi_{-}$is the splitting of a vector $\xi \in \mathbf{C}^{(n+1)^{2}}$ into its $\mathcal{N}_{0}, \mathcal{N}_{+}$and $\mathcal{N}_{-}$components and $\varrho_{+}, \varrho_{-}$stand for the restrictions of $\varrho$ to $\mathcal{N}_{+}$and $\mathcal{N}_{-}$, respectively, then

$$
\varrho(\xi, \xi)=\varrho_{-}\left(\xi_{-}, \xi_{-}\right)+\varrho_{+}\left(\xi_{+}, \xi_{+}\right)
$$

The subspaces $\mathcal{N}_{+}$and $\mathcal{N}_{-}$have dimensions $n(n+1) / 2$ and $n(n-1) / 2$ and are spanned by the vectors $s_{I J}$ and $d_{I J}$, respectively, where

$$
\left(s_{I J}\right)_{I I}=\left(s_{I J}\right)_{J J}=1,\left(s_{I J}\right)_{I J}=\left(s_{I J}\right)_{J I}=-1,\left(s_{I J}\right)_{i j}=0 \text { otherwise }(0 \leq I<J \leq n),
$$


and

$$
\begin{aligned}
\left(d_{I J}\right)_{I J}=\left(d_{I J}\right)_{0 I} & =\left(d_{I J}\right)_{J 0}=1,\left(d_{I J}\right)_{J I}=\left(d_{I J}\right)_{I 0}=\left(d_{I J}\right)_{0 J}=-1, \\
\left(d_{I J}\right)_{i j}= & 0 \text { otherwise }(1 \leq I<J \leq n) .
\end{aligned}
$$

For $n=2, \mathcal{N}_{-}$consists just of scalar multiples of $d_{12}$, and for $\alpha_{0}=0$ a rather laborious computation reveals that

$$
\begin{aligned}
& \varrho\left(d_{12}, d_{12}\right)=36 \frac{\left(1-\left|\alpha_{1}\right|^{2}\right)^{2}\left(1-\left|\alpha_{2}\right|^{2}\right)^{2}\left|1-\bar{\alpha}_{1} \alpha_{2}\right|^{4}}{\left|\alpha_{1}\right|^{2}\left|\alpha_{2}\right|^{2}\left|\alpha_{1}-\alpha_{2}\right|^{2}} \times \\
& \times\left[2\left(2-\left|\alpha_{1}\right|^{2}-\left|\alpha_{2}\right|^{2}\right)\left|1-\bar{\alpha}_{1} \alpha_{2}\right|^{2}+2\left(1-\left|\alpha_{1}\right|^{2}\right)\left(1-\left|\alpha_{2}\right|^{2}\right)+\left|\alpha_{1} \alpha_{2}\left(\alpha_{1}-\alpha_{2}\right)\right|^{2}\right]^{-2} \geq 0 ;
\end{aligned}
$$

so by Proposition 3.6 at least the quadratic form $\varrho_{-}$is positive definite for any $\alpha_{0}, \alpha_{1}, \alpha_{2} \in \mathbf{D}$.

Example. $n=2, \alpha_{0}=0, \alpha_{2}=-\alpha_{1}$. Denote $X=\left|\alpha_{1}\right|^{2}=\left|\alpha_{2}\right|^{2}$ and let $M$ be the $(3 \times 3)$ matrix of $\varrho_{+}$with respect to the basis $\left\{s_{I J}\right\}$ above. Then $M$ has eigenvalues $a r$, $a(s \pm \sqrt{t})$, where

$$
\begin{aligned}
a= & \frac{9\left(1-X^{2}\right)^{2}}{16 X^{4}\left(3-X^{2}\right)^{2}\left(1+X^{2}\right)^{2}}>0 \\
r= & 2 X\left(1+X^{2}\right)^{2}\left(7+2 X^{2}-X^{4}\right)>0 \\
s= & 9+34 X^{2}+48 X^{4}+6 X^{6}-X^{8}>0 \\
t= & 81+580 X^{2}+1860 X^{4}+3340 X^{6}+3046 X^{8} \\
& +668 X^{10}-284 X^{12}-108 X^{14}+33 X^{16}>0
\end{aligned}
$$

and

$$
s^{2}-t=32 X^{2}\left(1-X^{2}\right)^{2}\left(1+X^{2}\right)^{3}\left(1+4 X^{2}-X^{4}\right)>0 .
$$

In particular, $\varrho_{+}$is also positive definite. Thus the Green function for the operator $\Delta|G|^{-2} \Delta$, with $G$ the canonical divisor for a 2-point zero-set $\{\alpha,-\alpha\} \subset \mathbf{D}$, is positive.

ACKNOWLeDGements. The author's research was supported by GA AV ČR grants Nos. C1019601 and A1019701 and GA ČR grant No. 201/96/0411.

\section{REFERENCES}

1. S. Bochner, W. T. Martin, Several complex variables (Princeton University Press, Princeton, 1948).

2. P. L. Duren, D. Khavinson, H. S. Shapiro and C. Sundberg, Contractive zero-divisors in Bergman spaces, Pacific J. Math. 157 (1993) 37-56.

3. P. L. Duren, D. Khavinson, H.S. Shapiro and C. Sundberg, Invariant subspaces in Bergman spaces and the biharmonic equation, Michigan Math. J. 41 (1994) 247-259.

4. M. Engliš, A Loewner-type lemma for weighted biharmonic operators, Pacific J. Math. 179 (1997) 343-353.

5. M. Engliš and J. Peetre, A Green's function for the annulus, Ann. Mat. Pura. Appl. (IV) 171 (1996) 313-377. 
6. G. B. Folland, Introduction to partial differential equations. Second edition (Princeton University Press, Princeton, 1995).

7. P. R. Garabedian, A partial differential equation arising in conformal mapping, Pacific J. Math. 1 (1951), 485-524.

8. H. Hedenmalm, Open problems in the function theory of the Bergman space, in Festschrift in honour of Lennart Carleson and Yngve Domar (Proceedings of the conference held at Uppsala University, Uppsala, May 27-28, 1993) (Anders Vretblad, ed.), Acta Universitatis Upsaliensis, vol. 58, Uppsala Universitet (Uppsala, Sweden, 1995), 153-169.

9. H. Hedenmalm, A computation of Green functions for the weighted biharmonic operators $\Delta|z|^{-2 \alpha} \Delta$, with $\alpha>-1$, Duke Math. J. 75 (1994), 51-78.

10. H. Hedenmalm, Boundary value problems for weighted biharmonic operators, Algebra i Analiz 8 (1996), 173-192.

11. N. K. Nikolski1, Treatise on the shift operator. Spectral function theory (SpringerVerlag, 1986).

12. S. M. Shimorin, Factorization of analytic functions in weighted Bergman spaces, Algebra i Analiz 5 (1993), 155-177 (Russian); translation in St. Petersburg Math. J. 5 (1994), $1005-1022$.

13. S. M. Shimorin, On a family of conformally invariant operators, Algebra i Analiz 7 (1995), 133-158 (Russian); translation in St. Petersburg Math. J. 7 (1996), 287-306.

14. S. M. Shimorin, The Green function for the weighted biharmonic operator $\Delta\left(1-|z|^{2}\right)^{-\alpha} \Delta$ and the factorization of analytic functions, Zap. Nauchn. Sem. S.-Petersburg. Otdel. Mat. Inst. Steklov. (POMI) 222 (1995), 203-221. (Russian)

15. S. M. Shimorin, On the Green function for the weighted biharmonic operator $\Delta w^{-1} \Delta$ in the unit disc, Problems of Mathematical Analysis, to appear.

16. M. Stessin, An extension of a theorem of Hadamard and domination in the Bergman space, J. Funct. Anal 115 (1993) 212-226. 